\title{
Packing Rectangles into a Fixed Size Circular Container: Constructive and Metaheuristic Search Approaches
}

\author{
Mouaouia Cherif Bouzid ${ }^{1}$ and Said Salhi ${ }^{2}$ \\ ${ }^{1}$ LTI-Laboratory, Department of Logistics and Transport Engineering, ENST, Dergana, Algiers, Algeria. \\ ${ }^{2}$ Centre for Logistics \& Heuristic Optimisation, Kent Business School, The University of Kent, Canterbury, Kent \\ CT2 7PE, UK.
}

(3rd March 2020)

\begin{abstract}
We investigate the orthogonal packing of rectangular objects into a circular container of fixed radius. We propose a new constructive heuristic called pack which builds a feasible packing starting from an ordered list of rectangles. This decoding procedure is polynomial and permits to move from the permutations search space to the packings search space by means of simple combinatorial moves combined with powerful geometrical analytical forms. The pack procedure is integrated into two well known metaheuristics, namely, a variable neighbourhood search (VNS) and a simulated annealing (SA). Two variants, namely xVNS and xSA, which stand as accelerated versions of VNS and SA are also presented. The proposed methodology produces 32 new best solutions out of the 54 benchmark instances while requiring less computational effort than the state-of-the-art method. In addition, we conduct experiments on newly generated larger instances which we have made publicly available alongside their respective results obtained from the proposed metaheuristics.
\end{abstract}

Keywords: Rectangle packing, fixed size circular container, constructive heuristic, variable neighbourhood search, simulated annealing. []

\section{Introduction and literature review}

A packing problem can be defined as the optimisation of the choice and the location of a subset of objects into a container in such a way that no two objects overlap with each other and every object lies entirely within the container's perimeter while satisfying possible additional constraints. Several variants of the problem exist depending on features such as the dimensionality, the objects and container's shapes, the objective, etc (see Wäscher et al. (2007) for a typology of cutting and packing problems).

The complexity of packing problems have been tackled by several authors. For instance, $\mathrm{Li}$ and Cheng (1994) showed that deciding whether a set of squares can be packed into a larger rectangle is strongly NP-complete. Leung et al. (1990) also proved the same result when the target shape is a square.

\footnotetext{
Email addresses: m.cherif.bouzid@enst.dz (M.C. Bouzid, Corresponding author), s.salhi@kent.ac.uk (S. Salhi)
} 
Two decades later, Demaine et al. (2010) showed that deciding whether a given set of circles can be packed into a rectangle, an equilateral triangle, or a unit square are NP-hard problems. To the best of our knowledge, the complexity of deciding whether a set of rectangles can be packed into a circular container of fixed size has not been tackled yet in the literature.

Table 1 summarises the references that addressed the packing of rectangular objects into containers having either a circular or an arbitrary convex shape. The first column indicates the reference. The next four columns describe the features of the problem considered while the last two columns indicate eventual additional constraints and the method adopted by the authors. Two main applications arise, one in the timber industry (Hinostroza et al. 2013) and the other in the satellite module layout design denoted SMLD (see for instance Zhong et al. (2019)). The latter consists in packing, inside a satellite module, a set of weighted objects on one or two bearing plates having a circular shape while optimising the inertia performance of the whole system and respecting some additional constraints such as ensuring a minimal equilibrium. This problem, which can be considered as a sophisticated multi-level two-dimensional packing problem where each level corresponds to a packing problem into a circular container, received some attention in the last two decades. Starting with Feng et al. (1999), the authors proposed to solve the problem by means of graph theory, group theory and global optimisation. Later, Teng et al. (2001) adopted a two-phases heuristic method which first solves a two-dimensional packing problem then translates bearing plates to improve the centre of mass of the satellite. Also, Sun and Teng (2003) proposed a two-phases heuristic based on non-linear programming which first distributes the objects on the bearing plates by means of a genetic algorithm and then solves several two-dimensional packing problems using ant colony optimisation. Xu et al. (2007) designed a particle swarm-based method while Liu and Teng (2008) put forward an evolutionary algorithm with expert knowledge. The latter formulated the problem as a non-linear program where the violation of the constraints is penalised into the objective function. Wang and Teng (2009) adopted a similar approach in a slightly different problem. Zhang et al. (2008) proposed a two-phases heuristic while $\mathrm{Xu}$ et al. (2010) designed a constructive heuristic based on an ordering of rectangles which is then embedded into a genetic algorithm. An interesting heuristic approach which relies heavily on physics notions such as energy and forces is put forward by Li et al. (2014). Their heuristic is applied on five SMLD examples with interesting results. Zhao et al. (2014) tackled the problem using particle swarm optimisation and immune algorithms with expert knowledge while Li et al. (2016) proposed an approach based on ant colony and particle swarm optimisation with expert knowledge. Fakoor et al. (2017) considered an extension of SMLD that limits the resonance phenomenon due to interferences while packing the objects into the module. Their approach relies on particle swarm optimisation and gradient-based sequential quadratic programming. Zhong et al. (2019) also extended SMLD by forbidding a circular region at the origin of the plates which represents a standing column. 
Their heuristic is based on two main parts, namely, the assignment/reassignment of components to various bearing plates, and the layout optimisation which places the components on the surfaces using a differential evolution algorithm.

An interesting application of packing rectangles into a circle without rotation is found in the timber industry where the cutting of boards from logs takes place. Hinostroza et al. (2013) considered this problem and formulated it as a mixed-integer non-linear program (MINLP). The exact resolution of that formulation using a commercial solver turned out to be limited to instances involving 9 rectangles at most. The authors proposed an ordering heuristic and a simulating annealing (SA) to tackle larger instances. This SA algorithm will be revisited in this paper as this forms a suitable basis for benchmarking purposes.

When it comes to the packing of objects into containers having an arbitrary convex shape, the resolution is usually based on non-linear programming and the use of a generic solver. Birgin et al. (2006a) proposed a general approach based on so-called "sentinel sets" and non-linear programming for packing objects into an Euclidean $n$-dimensional space. The authors restricted their approach to address the two-dimensional packing of rectangles and rectangle-likes objects into arbitrary convex regions. Using a solver, they could pack up to only 40 objects. Birgin et al. (2006b) tackled a less general problem formulated as a non-linear program using a solver in a multi-start fashion where they were able to pack up to 64 rectangles in over 2 hours on an AMD Opteron 244/1.8 GHz. Birgin and Lobato (2010) considered the problem of packing a maximum number of equal rectangles into an arbitrary convex region while allowing a common rotation angle for all objects and a $90^{\circ}$ rotation for some of them. They formulated the problem as a MINLP using a branch \& bound technique coupled with active set strategies. Similarly to Birgin et al. (2006b), Cassioli and Locatelli (2011) formulated the orthogonal packing of identical rectangle into a convex region as an unconstrained mixed integer global optimisation problem and solved it using an iterated local search combined with the L-BFGS algorithm of Liu and Nocedal (1989).

López and Beasley (2018b) considered the orthogonal packing of unequal rectangles into a circular container of fixed size. They formulated the problem as a mixed integer non-linear program and solved it using a third party solver, called SCIP, which is limited to solving small instances only. As we address the same problem, we will revisit their formulation in the next section. The authors then proposed an interesting implementation of formulation search space algorithm (FSS) to solve the problem which relies heavily on the use of SCIP. Instances involving up to 30 rectangles were generated with interesting results though requiring a relatively large amount of CPU. FSS is an interesting concept designed by Mladenović et al. (2005) where the change in neighbourhoods is replaced by the formulation space neighbourhood instead. For more informations on non-linear programming and packing problems, the recent review by Birgin (2016) makes an interesting and informative read. 


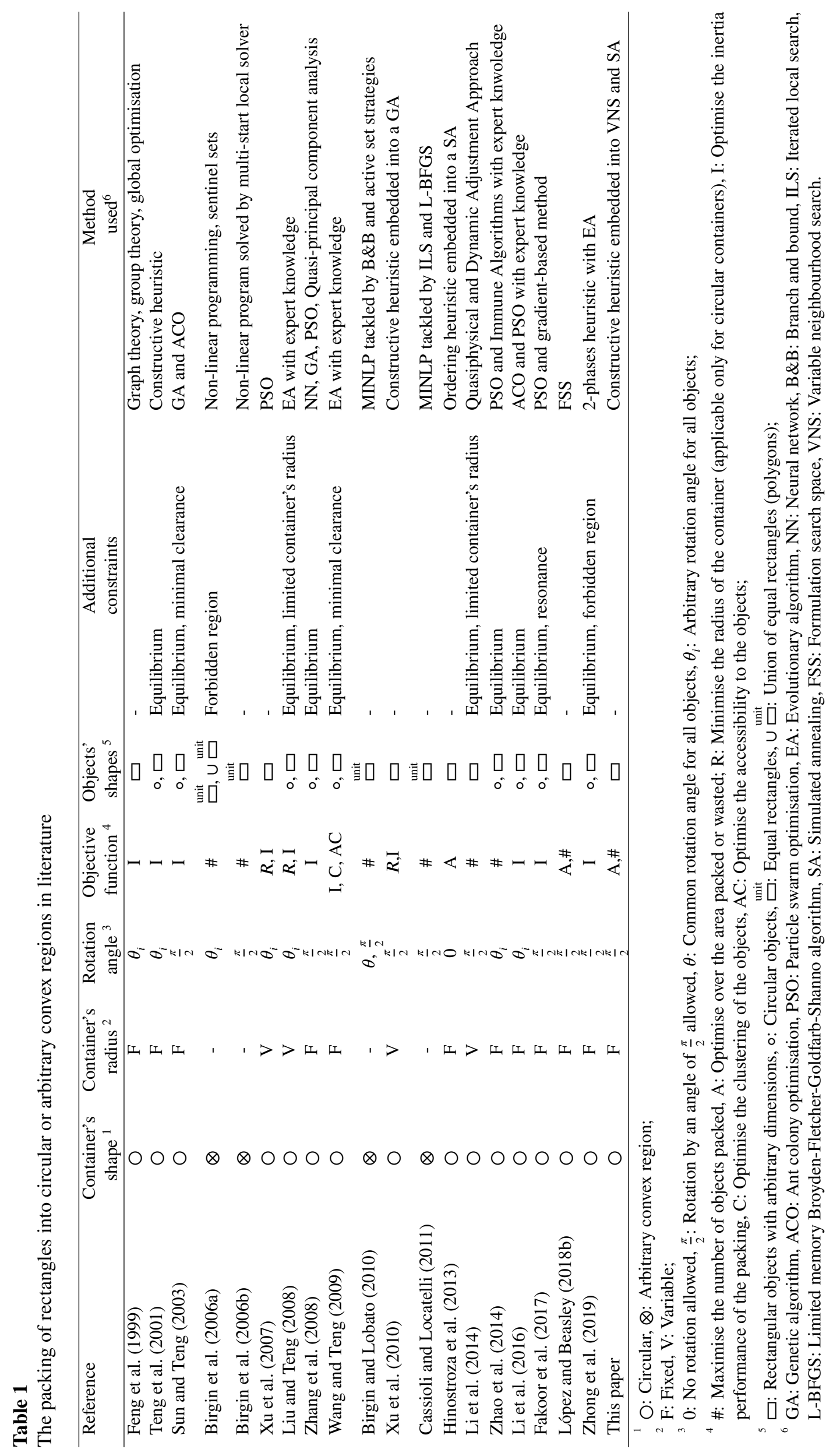


The above literature review suggests that, even though non-linear programming-based approaches developed for packing problems allow many features (arbitrary convex container, rectangular-like objects, arbitrary or uniform rotation, forbidden regions, etc), they still suffer from limitations due to the prohibiting computing time they require. Moreover, it is worth mentioning that in the presented literature, heuristic approaches which take benefit of the underlying combinatorial aspects of the considered packing problems have been seldom explored.

As mentioned in Table 1, this study addresses the orthogonal packing of unequal rectangles into a circular container of fixed size with the objective of maximising either the number of objects or the total area packed. To the best of our knowledge, the only reference that tackled this specific problem is due to López and Beasley (2018b). Given the two-folds complexity of a solution structure which is to determine the subset of rectangles to pack and their locations, we introduce a decoding procedure called pack which receives initially an ordered list of rectangles then returns a feasible packing. This combinatorial heuristic permits to move from the permutations search space to the packings search space. It is based on the concept of "border" that we introduce, a number of initial configurations which indicate how to pack the first objects in the list, and several moves which allow the packing of new objects. As the use of the constructive mechanism of pack on a single list is unlikely to provide a global optimum, we propose to integrate this procedure into two powerful metaheuristics, namely, a variable neighbourhood search and a simulated annealing. Moreover, exploiting the past placements of some items in the search permit us to propose two accelerated variants of those metaheuristics. To assess the performance of the proposed methodology, experiments are conducted on two datasets, one being a literature benchmark and the other which we newly generated and made publicly available for further benchmarking.

The contributions of this study are:

- to address the orthogonal packing of unequal rectangles into a circular fixed size container;

- to propose and determine the complexity of a flexible and efficient constructive heuristic 'pack';

- to provide and justify an algorithm 'buildBorder' which determines the surrounding border of an already known packing;

- to efficiently integrate pack into a variable neighbourhood search and a simulated annealing and to provide two accelerated version by means of buildBorder;

- to show the superiority of our methodology against the state-of-the-art method using benchmark instances and to assess the performance of the proposed metaheuristics on a new dataset that we generate and made publicly available.

The remainder of the paper is organised as follows: the next section is devoted to the detailed presentation of the procedure pack. In Section 3, the integration of pack into VNS and SA schemes is described. 
Section 4 is dedicated to computational experiments including the generation of the newly constructed datasets. Finally, conclusions and some suggestions are given in Section 5.

\section{The procedure pack}

In this section, we first define formally the packing problem and we recall the formulation proposed by López and Beasley (2018b). We then introduce a decoding procedure which we refer to as 'pack'. The time complexity of the procedure 'pack' is also determined here. Finally, we present an algorithm to construct the border surrounding an already known packing.

\subsection{Problem definition and formulation}

Let $[n]=\{1, \ldots, n\}$ be a set of rectangular objects of dimensions $\left(L_{i}, W_{i}\right)_{i \in[n]}$ where $L_{i}$ and $W_{i}$ denote the horizontal and vertical lengths of the rectangles respectively. Let $v_{i}$ be a value associated with rectangle $i$ and assume a container having a circular shape of fixed radius $R$. We define a packing $p$ as the placement of a subset of rectangles into the container such that no two rectangles overlap and no rectangle passes through the container's perimeter. Formally, $p$ is a 4-tuple $\left(\alpha_{i}, x_{i}, y_{i}, \theta_{i}\right)_{i \in[n]}$ such that $\alpha_{i}$ equals 1 if the rectangle $i$ is packed and 0 otherwise, $\left(x_{i}, y_{i}\right)$ are the coordinates of the centre of rectangle $i$ and $\theta_{i}$ its rotational angle which value is considered in this study to be either 0 or $\frac{\pi}{2}$. In case rectangle $i$ is not packed, $\left(x_{i}, y_{i}\right)$ and $\theta_{i}$ are null. Let $V_{i}^{1}=\left(x_{i}+L_{i} / 2, y_{i}+W_{i} / 2\right), V_{i}^{2}=\left(x_{i}-L_{i} / 2, y_{i}+W_{i} / 2\right)$, $V_{i}^{3}=\left(x_{i}-L_{i} / 2, y_{i}-W_{i} / 2\right)$ and $V_{i}^{4}=\left(x_{i}+L_{i} / 2, y_{i}-W_{i} / 2\right)$ be the coordinates of the upper-right, upper-left, lower-left and lower-right vertices of rectangle $i$ respectively.

Some definitions related to the connectedness of a packing:

- Two different rectangles $i$ and $j$ overlap if $\left|x_{i}-x_{j}\right|-\left(L_{i}+L_{j}\right) / 2<0$ and $\left|y_{i}-y_{j}\right|-\left(W_{i}+W_{j}\right) / 2<0$.

- The sides $A B$ and $C D$ of two rectangles are contiguous if $A B$ and $C D$ are colinear and $C \in A B$, $D \in A B$ or $A \in C D$

- A partial packing $p$ is a 4-tuple $p=\left(\alpha_{i}, x_{i}, y_{i}, \theta_{i}\right)_{i \in E}$ such that $E \subsetneq[n]$ and $|E|>0$.

- A partial packing $p$ is empty if $\alpha_{i}=0$ for all $i$ in $E$.

- Two partial packings $p_{1}=\left(\alpha_{i}, x_{i}, y_{i}, \theta_{i}\right)_{i \in E_{1}}$ and $p_{2}=\left(\alpha_{i}, x_{i}, y_{i}, \theta_{i}\right)_{i \in E_{2}}$ are disjoints if $E_{1} \cap E_{2}=\emptyset$.

- Two disjoints partial packings $p_{1}$ and $p_{2}$ overlap if there exists a rectangle packed in $p_{1}$ and another one packed in $p_{2}$ such that the two rectangles overlap.

- Two partial packings are compatible if they are disjoints and do not overlap.

- The union of two compatible partial packings $p_{1}$ and $p_{2}$ is defined by $p_{1} \cup p_{2}=\left(\alpha_{i}, x_{i}, y_{i}, \theta_{i}\right)_{i \in E_{1} \cup E_{2}}$. 
- Two compatible partial packings $p_{1}$ and $p_{2}$ are contiguous if a rectangle packed in $p_{1}$ and a rectangle packed in $p_{2}$ have contiguous sides.

- A packing $p$ is said to be connected if there exists no two non-empty compatible partial packings which are not contiguous and which union equals $p$.

The quality of a packing $p$ can be measured by $f(p)=\sum_{i \in[n]} v_{i} \alpha_{i}$ as follows:

(i) if $v_{i}=L_{i} W_{i}$ then $f(p)$ corresponds to the total area packed and,

(ii) if $v_{i}=1$ then $f(p)$ becomes the total number of rectangles packed.

The packing problem considered in this study is to find a packing $p^{*}$ having a maximum value $f\left(p^{*}\right)$.

López and Beasley (2018b) formulated the problem of packing $n$ rectangles of dimensions $\left(L_{i}, W_{i}\right)_{i \in[n]}$ into a circular container of fixed radius $R$ without rotation as follows:

$$
\begin{aligned}
& \text { (P) } \quad \max \sum_{i=1}^{n} v_{i} \alpha_{i} \\
& \text { s.t. } \\
& \quad-\alpha_{i}\left(\sqrt{R^{2}-W_{i}^{2} / 4}-L_{i} / 2\right) \leq x_{i} \leq \alpha_{i}\left(\sqrt{R^{2}-W_{i}^{2} / 4}-L_{i} / 2\right), i=1, \ldots, n \\
& \quad-\alpha_{i}\left(\sqrt{R^{2}-L_{i}^{2} / 4}-W_{i} / 2\right) \leq y_{i} \leq \alpha_{i}\left(\sqrt{R^{2}-L_{i}^{2} / 4}-W_{i} / 2\right), i=1, \ldots, n \\
& \quad\left\|V_{i}^{k}\right\|^{2} \leq \alpha_{i} R^{2}+\left(1-\alpha_{i}\right) R_{i}^{2}, \quad i=1, \ldots, n ; k=1, \ldots, 4 \\
& \quad \alpha_{i} \alpha_{j}\left[\max \left\{\left|x_{i}-x_{j}\right|-\left(L_{i}+L_{j}\right) / 2,\left|y_{i}-y_{j}\right|-\left(W_{i}+W_{j}\right) / 2\right\}\right] \geq 0, \\
& \quad i=1, \ldots, n ; j=1, \ldots, n ; j>i \\
& \quad \alpha_{i} \in\{0,1\}, i=1, \ldots, n
\end{aligned}
$$

where $\left\|V_{i}^{k}\right\|$ is the euclidean norm of $V_{i}^{k}$ and $R_{i}$ is the radius of the smallest circle encompassing the $i$-th rectangle in case it is positioned at the origin. In the formulation $(P)$, depending on the value of $v_{i},(1)$ maximises either the total area or the total number of rectangles packed. Constraints (2)-(3) make sure that in case a rectangle is packed, its coordinates lie in the appropriate range, otherwise its coordinates are forced to be zero. Constraints (4) ensure that, in case rectangle $i$ is packed, all its vertices lie within the perimeter of the container. Constraints (5) prevent the overlapping between rectangles that are packed. The binary nature of the variables $\alpha_{i}$ is guaranteed by the constraints (6). Even though this formulation does not consider rotations, the authors proposed an elegant extension to allow orthogonal rotations of the rectangles. Suppose that a rectangle $i$ can be rotated by a right angle, it is sufficient to add a new rectangle $j$ that represents rectangle $i$ if it is rotated, so that $L_{j}=W_{i}, W_{j}=L_{i}, v_{j}=v_{i}$ then, to add to the formulation: 


$$
\alpha_{i}+\alpha_{j} \leq 1
$$

Constraint (7) ensures that not both the original rectangle and its rotated equivalent are used. The authors used the solver SCIP to tackle $(P)$ but could not solve any proposed instance to proven global optimality within the time limit imposed.

The number of solutions in the considered problem is dramatically large as any solution requires to identify which subset of rectangles to pack and also where to pack them. The first decision involves $\sum_{i=1}^{n}\left(\begin{array}{l}n \\ i\end{array}\right)=O\left(2^{n}\right)$ possibilities while the second offers an infinite number of choices given the decision is made over a subset of $\mathbb{R}^{2}$. The main idea of our approach is to consider a much smaller search space which consists of all the ordered lists of the $n$ rectangles that we will simply call lists. This is possible with a "decoding" procedure which permits to move from the lists search space to the packings search space.

\subsection{Overview of the procedure pack}

The procedure pack makes an intensive use of the notion of "border". The border associated with a packing $p$ is a data structure, preferably implemented as a circular chained list, which contains the corner points of the rectangles that fall on the external side of $p$. This array is updated during the procedure by adding and removing corner points whenever a new rectangle is packed. To avoid redundancy, the handling of a border follows two rules:

(i) the border must not contain any sequence of three aligned points and,

(ii) any two successive points in the border must be different (see for instance Figure 1).

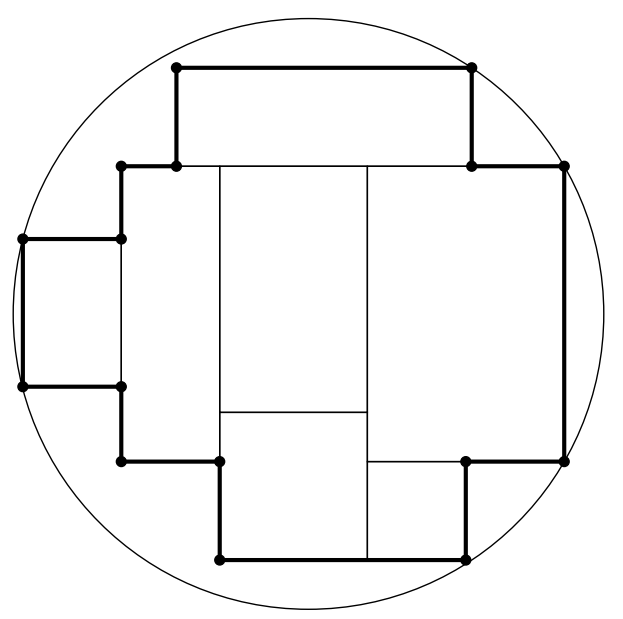

Fig. 1. Example of a packing delimited by its border

The procedure pack (see Algorithm11) requires as input a list $l$ and returns a packing $p_{\text {best }}$. Initially, no rectangle is packed in $p_{\text {best }}$ (see line 1). The quality of the packing obtained after applying the procedure 
pack on a list depends highly on the way the first one or two objects are placed into the container. Therefore, we apply several initial configurations to maximise the chances of obtaining a good packing. For each possible initial configuration, an initial packing is built and its border is initialised (see line 4). Next, for every remaining rectangle in the list, a promising feasible placement is sought around the border among all possible placements (see line 6). The determination of a promising feasible placement is presented in Section 2.4. If such a placement exists, the incumbent rectangle is packed at that position and the border is updated (see line 8). Then, the procedure continues with the next rectangle in the list (see line 10p. When the end of the list is reached, if the incumbent packing is better than the best one, the best packing is updated (see line 12). The procedure terminates when all initial configurations have been considered and the best packing is returned.

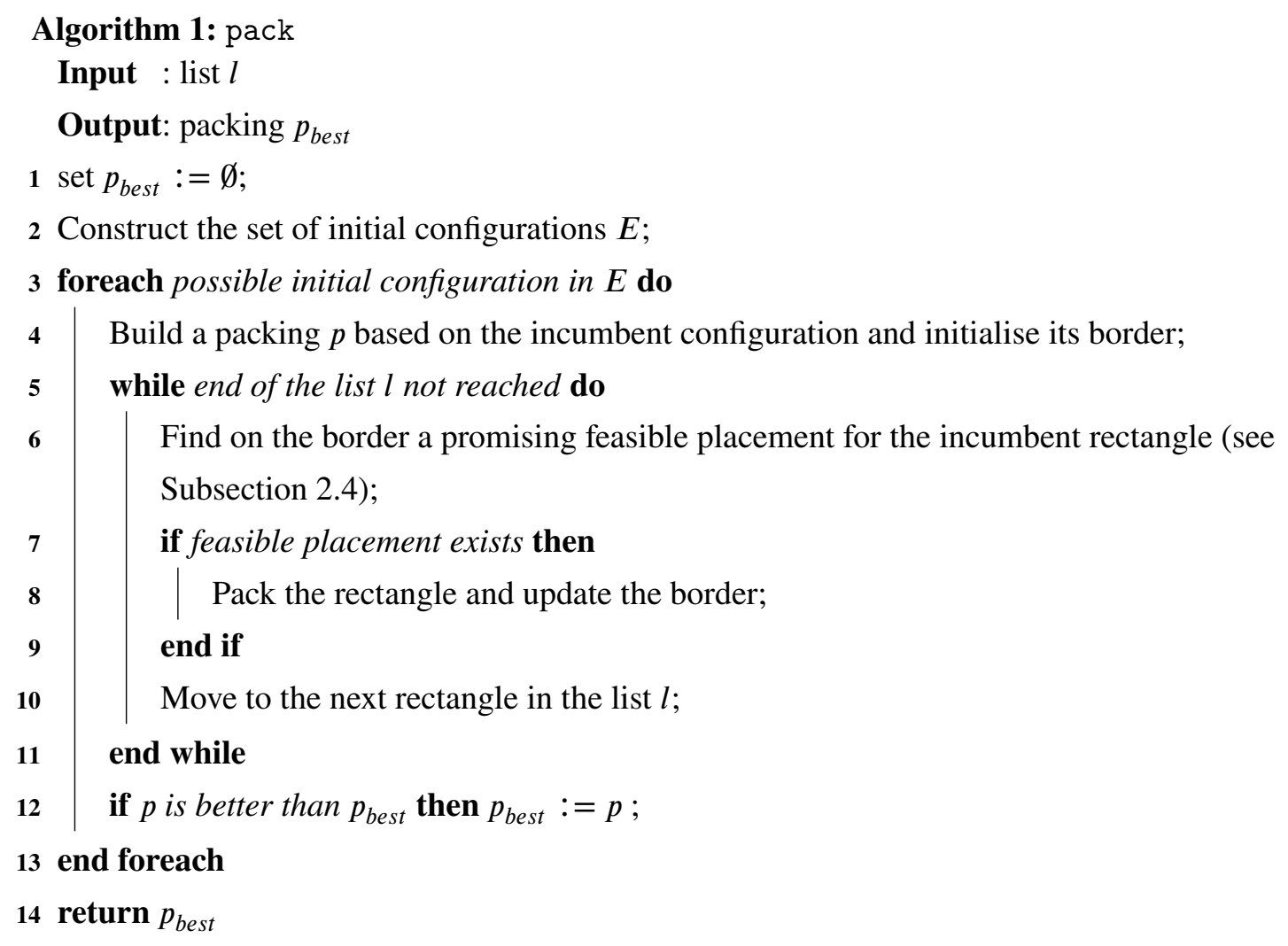

\subsection{Initial configurations (step 2 of Algorithm 1 )}

We apply five initial configurations denoted by C (centre), R (right), T (top), RS (right-shifted), RA (right-aligned). We describe the initial configurations, the way to calculate the coordinates of the objects they pack and the composition of the initial border. For the sake of clarity, assume without loss of generality that the first two rectangles in the list are indexed 1 and 2 .

In the $\mathrm{C}$ configuration, the centre of Rectangle 1 is simply placed at the origin. In the $\mathrm{R}$ configuration, Rectangle 1 is placed in such a way that its lower right and upper right corners lie on the circle. In this case, the centre $(x, y)$ of Rectangle 1 must satisfy the following system $\left(S_{1}\right)$ : 


$$
\left(S_{1}\right)\left\{\begin{array}{lll}
\left(x+\frac{L}{2}\right)^{2}+\left(y-\frac{W}{2}\right)^{2}=R^{2} & \ldots & \text { (lower-right corner on the circle) } \\
\left(x+\frac{L}{2}\right)^{2}+\left(y+\frac{W}{2}\right)^{2}=R^{2} & \ldots & \text { (upper-right corner on the circle) } \\
x \geq 0 & \ldots & \text { (rectangle must fit into the circle) }
\end{array}\right.
$$

The solution of $\left(S_{1}\right)$ is $(x, y)=\left(\sqrt{R^{2}-\frac{W^{2}}{4}}-\frac{L}{2}, 0\right)$. This configuration is applicable only if $R^{2}-\frac{W^{2}}{4} \geq 0$.

In the $\mathrm{T}$ configuration, Rectangle 1 is placed in such a way that its upper right and upper left corners are on the circle. Applying similar calculations to the ones applied for $\mathrm{R}$ configuration results in the coordinates $\left(0, \sqrt{R^{2}-\frac{L^{2}}{4}}-\frac{W}{2}\right)$.

In the three configurations $\mathrm{C}, \mathrm{R}$ and $\mathrm{T}$, the initial border is given by $\left(V_{1}^{1}, V_{1}^{2}, V_{1}^{3}, V_{1}^{4}\right)$.

In the RS configuration, Rectangle 1 is first placed as in the R one. Rectangle 2 is put on the left of the first one then shifted upward in a way that the upper-left corner of the second rectangle lies on the circle (see Figure 2). Let $\left(x_{1}, y_{1}\right)$ and $(x, y)$ be the coordinates of Rectangles 1 and 2 respectively. The location of Rectangle 1 is known a priori by the $\mathrm{R}$ configuration. Thus, $(x, y)$ must satisfy the following system:

$$
\left\{\begin{array}{llr}
\left(x_{1}-\frac{L_{1}}{2}-L_{2}\right)^{2}+\left(y+\frac{W_{2}}{2}\right)^{2}=R^{2} & \ldots & \text { (upper-left corner of Rectangle } 2 \text { on the circle) } \\
y \geq 0 & \ldots & \text { (Rectangle } 2 \text { must fit into the circle) }
\end{array}\right.
$$

leading to $(x, y)=\left(x_{1}-\frac{L_{1}}{2}-\frac{L_{2}}{2}, \sqrt{R^{2}-\left(x_{1}-\frac{L_{1}}{2}-L_{2}\right)^{2}}-\frac{W_{2}}{2}\right)$. This placement is feasible only if $R^{2}-\left(x_{1}-\frac{L_{1}}{2}-L_{2}\right)^{2} \geq 0$. To ensure that the packing remains connected, the condition $y \leq \frac{W_{1}+W_{2}}{2}$ is added. The border is defined by $\left(V_{1}^{1}, V_{1}^{2}, V_{2}^{1}, V_{2}^{2}, V_{2}^{3}, V_{2}^{4}, V_{1}^{3}, V_{1}^{4}\right)$ on condition that $V_{1}^{2} \neq V_{2}^{1}$ and $V_{2}^{4} \neq V_{1}^{3}$, in which case, the redundant nodes are omitted.

In the RA configuration, Rectangle 1 is placed on Rectangle 2 in such a way that their left sides are

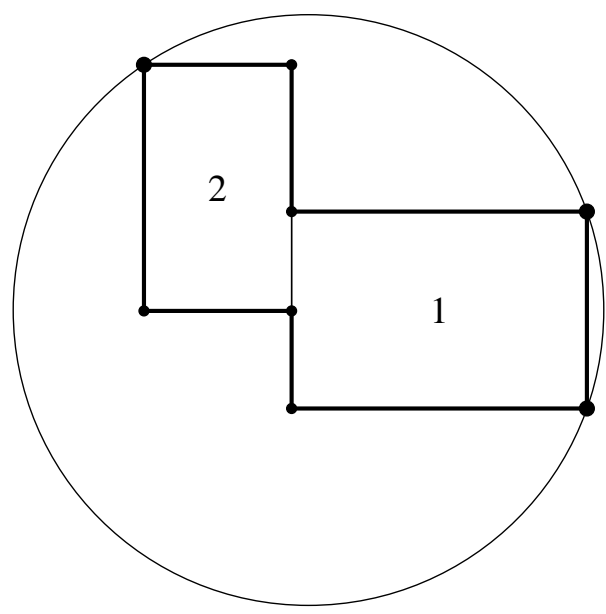

Fig. 2. Illustration of the RS initial configuration and its border 
aligned and, the upper-right corner of Rectangle 1 and the lower-right corner of Rectangle 2 lie on the circle (see Figure 3). The coordinates $(x, y)$ of the centre of Rectangle 1 must satisfy the following set of conditions $\left(S_{2}\right)$.

$\left(S_{2}\right)\left\{\begin{array}{lrr}\left(x+\frac{L_{1}}{2}\right)^{2}+\left(y+\frac{W_{1}}{2}\right)^{2}=R^{2} & \ldots & \text { (upper-right corner of Rectangle } 1 \text { on the circle) } \\ \left(x-\frac{L_{1}}{2}+L_{2}\right)^{2}+\left(y-\frac{W_{1}}{2}-W_{2}\right)^{2}=R^{2} & \ldots & \text { (lower-right corner of Rectangle } 2 \text { on the circle) } \\ x \geq 0, y \geq 0 & \ldots & \text { (Rectangle 1 must fit into the circle) }\end{array}\right.$

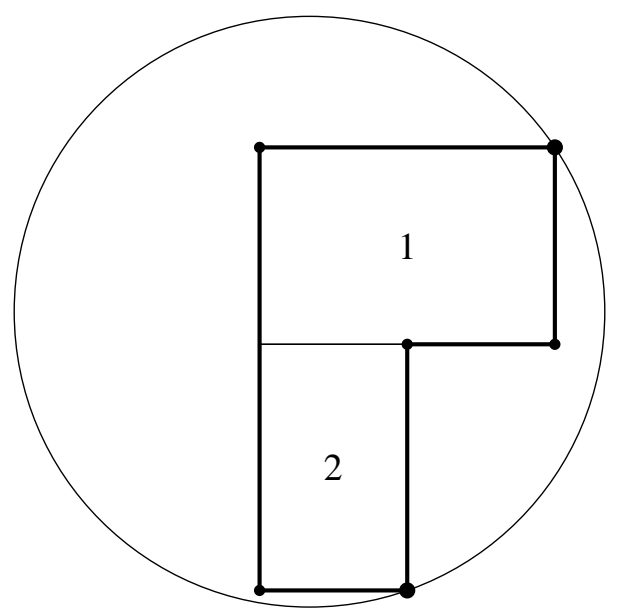

Fig. 3. Illustration of the RA initial configuration and its border

The system $\left(S_{2}\right)$ consists of two bivariate polynomials of second degree. The problem of solving a system of multivariate quadratic equations over finite fields is NP-complete (Garey and Johnson, 1979). However, the resolution of $\left(S_{2}\right)$ is possible given its small dimensions using the Buchberger's algorithm (Buchberger. 1976). Building over the initial set of polynomials, this algorithm constructs an equivalent system of polynomials in the form of a Gröbner basis which is usually easier to solve than the original system. Making use of the SageMath system for symbolic calculus (SageMath, Inc. 2018), the whole process results in the following solution

$$
\begin{aligned}
& x=-\frac{1}{2} L_{2}+\frac{1}{2}\left(W_{1}+W_{2}\right) \sqrt{\frac{4 R^{2}}{\left(L_{1}-L_{2}\right)^{2}+\left(W_{1}+W_{2}\right)^{2}}-1} \\
& y=\frac{1}{2} W_{2}-\frac{1}{2}\left(L_{1}-L_{2}\right) \sqrt{\frac{4 R^{2}}{\left(L_{1}-L_{2}\right)^{2}+\left(W_{1}+W_{2}\right)^{2}}-1}
\end{aligned}
$$

The coordinates of rectangle 2 are then $\left(x-\frac{L_{1}}{2}+\frac{L_{2}}{2}, y-\frac{W_{1}}{2}-\frac{W_{2}}{2}\right)$.

Obviously, the RA configuration is applicable only if $\left(\frac{L_{1}}{2}-\frac{L_{2}}{2}\right)^{2}+\left(\frac{W_{1}}{2}+\frac{W_{2}}{2}\right)^{2} \leq R^{2}$. If the two rectangles have different widths then the border is $\left(V_{1}^{1}, V_{1}^{2}, V_{2}^{3}, V_{2}^{4}, V_{2}^{1}, V_{1}^{4}\right)$. It is worth mentioning that if Rectangles 1 and 2 have the same widths and their stacking is considered as one rectangle then the formula reduces to the one obtained for the $\mathrm{R}$ configuration. Also, as Rectangles 1 and 2 are contiguous in this configuration, the resulting packing is connected. 


\subsection{Determining a promising feasible placement (step 6 of Algorithm 1 )}

A placement of the incumbent rectangle is feasible if no overlapping occurs between this rectangle and a rectangle already packed and no over-crossing happens with the container's perimeter. We determine a promising feasible placement for the incumbent rectangle around the border as follows. Let $A, B$ and $C$ be three consecutive corner points on the border. As no redundancy is allowed, the three points are disjoints and not aligned. We consider five cases when placing the incumbent rectangle on the border. The dashed area in Figure 4 represents the inner side of the border. The case (a) is when the point $C$ lies on the right side of the line $(A B)$ in which case the incumbent rectangle is stuck to the corner point $B$. When $C$ is on the left side of $(A B)$, we consider two possible placements of the rectangle: either it sticks to the edge segment $A B$ (denoted case (b)), or, it sticks to the edge segment $B C$ (denoted case (c)). If the rectangle overlaps with the container in cases (b) or (c), the rectangle is translated according to $\overrightarrow{B A}$ until its upper right corner lies on the circle (denoted case (d)), or, it is translated according to $\overrightarrow{B C}$ until its upper right corner lies on the circle (denoted case (e)) respectively. All these placements are considered for every corner point on the border. Among all these placements, the feasible placement having a minimum distance to the centre of the container is returned. The rectangle is packed if and only if such a feasible placement exists.

(a)

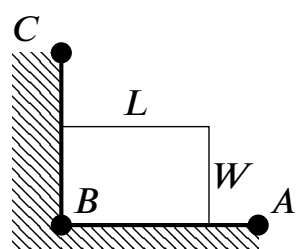

(b)

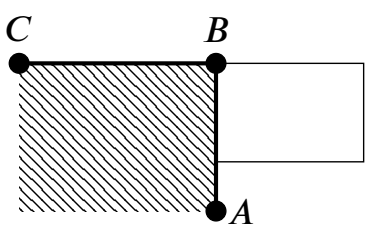

(c)

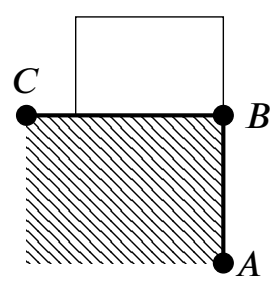

(d)

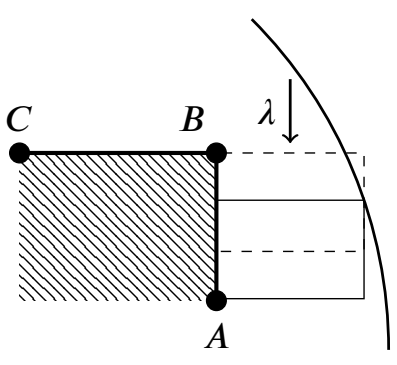

(e)

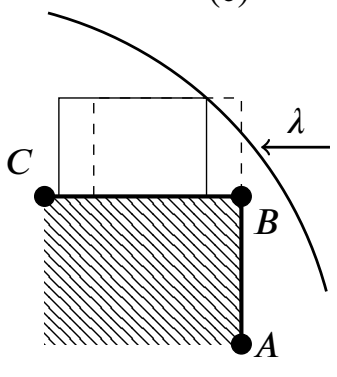

Fig. 4. Five ways of placing a rectangle on the border.

In order to determine whether the point $C$ lies on the right or left side of $(A B)$, we test the sign of the dot product $N_{(A B)} \cdot \overrightarrow{B C}$ where $N_{(A B)}$ is the normal vector to the line $(A B) . N_{(A B)}$ is given by the gradient of the equation of $(A B)$ that is $N_{(A B)}=\left(y_{A}-y_{B}, x_{B}-x_{A}\right)$. It is easy to see that:

$$
N_{(A B)}=\left[\begin{array}{rr}
0 & -1 \\
1 & 0
\end{array}\right] \cdot \overrightarrow{A B}
$$

Therefore, $N_{(A B)}$ points always to the left side of $(A B)$, precisely the inner side of the border. As a result, 
if the dot product $N_{(A B)} \cdot \overrightarrow{B C}$ is negative then $N_{(A B)}$ and $\overrightarrow{B C}$ are pointing in opposite directions which corresponds to case (a). If this product is positive then it corresponds to the remaining cases.

Let $\left(x_{i}, y_{i}\right)_{i=a, \ldots, e}$ be the coordinates of the centre of the incumbent rectangle for cases (a)-(e) respectively. Using basic mathematical manipulations, we get the following:

$$
\begin{aligned}
& \left(x_{a}, y_{a}\right)=B+\frac{L}{2\|\overrightarrow{B A}\|} \overrightarrow{B A}+\frac{W}{2\|\overrightarrow{B C}\|} \overrightarrow{B C} \\
& \left(x_{b}, y_{b}\right)=B+\frac{W}{2\|\overrightarrow{B A}\|} \overrightarrow{B A}+\frac{L}{2\|\overrightarrow{C B}\|} \overrightarrow{C B} \\
& \left(x_{c}, y_{c}\right)=B+\frac{L}{2\|\overrightarrow{B C}\|} \overrightarrow{B C}+\frac{W}{2\|\overrightarrow{A B}\|} \overrightarrow{A B}
\end{aligned}
$$

Regarding the cases (d) and (e), the problem is to find a step size $\lambda$ which satisfies:

$$
\begin{aligned}
& \left(\left\|B+\lambda \frac{\overrightarrow{B A}}{\|\overrightarrow{B A}\|}+L \frac{\overrightarrow{C B}}{\|\overrightarrow{C B}\|}\right\|\right)^{2}=R^{2} \quad \text { (case (d)) } \\
& \left(\left\|B+\lambda \frac{\overrightarrow{B C}}{\|\overrightarrow{B C}\|}+W \frac{\overrightarrow{A B}}{\|\overrightarrow{A B}\|}\right\|\right)^{2}=R^{2} \quad \text { (case (e)) }
\end{aligned}
$$

In case $(d)$, in addition to $(11), \lambda$ must satisfy the following two conditions in order to maintain the connectedness of the packing:

$$
\begin{aligned}
& \lambda>0 \Rightarrow \lambda \leq\|\overrightarrow{B A}\| \\
& \lambda<0 \Rightarrow \quad|\lambda| \leq W
\end{aligned}
$$

Whatever the sense in which the rectangle is shifted, the conditions (13) and (14) ensure that it remains contiguous to the edge $B A$. Similarly, in case (e), in addition to $[12$, the following two conditions must hold:

$$
\begin{aligned}
& \lambda>0 \Rightarrow \lambda \leq\|\overrightarrow{B C}\| \\
& \lambda<0 \Rightarrow \quad|\lambda| \leq L
\end{aligned}
$$

where the conditions (15) and (16) ensure the rectangle remains contiguous to the edge $B C$.

The expressions (11) and (12) are second degree equations in $\lambda$. If no overlap occurs, the smallest root in absolute value, when the discriminant is non negative, is chosen in order to keep the rectangle inside the container.

In all cases (a)-(e), if the line $(A B)$ is horizontal in spite of being vertical or vice-versa, it is sufficient to swap the two values $L$ and $W$ in all the expressions (8)-(12), (14) and (16).

As described above, all the initial configurations and the moves used for the placements of the rectangles in the procedure pack() are designed in such a way that the resulting packing is connected as it is 
stated in the following proposition.

Proposition 1. pack returns a connected packing.

\subsection{Updating the border (step 8 of Algorithm 1 )}

When a rectangle is packed on a border, the latter needs to be updated. Assume that $A, B$ and $C$ are in the first quadrant of the plane and that $i$ is the index of the incumbent rectangle. The border update for the other possibilities can be deduced from the following descriptions. In case (a), the sequence $\left(V_{i}^{4}, V_{i}^{1}, V_{i}^{2}\right)$ is inserted in place of the corner point $B$ in the border. In case (b) (resp. (e)), the sequence $\left(V_{i}^{3}, V_{i}^{4}, V_{i}^{1}\right)$ (resp. $\left.\left(V_{i}^{3}, V_{i}^{4}, V_{i}^{1}, V_{i}^{2}\right)\right)$ replaces (resp. precedes) $B$ in the border, otherwise, in case (c) (resp. (d)), the sequence $\left(V_{i}^{1}, V_{i}^{2}, V_{i}^{3}\right)$ (resp. $\left.\left(V_{i}^{4}, V_{i}^{1}, V_{i}^{2}, V_{i}^{3}\right)\right)$ replaces (resp. succeeds) $B$ in the border. Once the rectangle is packed, a post-processing is operated on the border to keep the corner points only.

\subsection{Flexibility of the procedure pack}

Applying small changes can broaden the applicability of pack considerably and, consequently the applicability of our methodology. As an example, the following two possible extensions to pack can be easily implemented.

(a) Enable the rotation of rectangles by $90^{\circ}$ - In case the considered instance allows the rotation of rectangles by $90^{\circ}$, any rectangle for which no feasible placement could be found is rotated by $90^{\circ}$ and a feasible placement is sought. If the latter exists, the rotated rectangle is packed. Otherwise, the procedure pack moves to the next rectangle.

(b) Forbid a region - It is possible to forbid a connected region during the packing process. To do so, the undesired region is simply considered as an initial configuration defined by a border in our procedure. Then, the procedure packs the rectangles around the border using the moves described above.

\subsection{Complexity of pack}

The following result gives the complexity of pack.

Theorem 1. pack runs in $O\left(k n^{2}\right)$ where $k$ is the number of initial configurations and $n$ the number of rectangles.

Proof. The complexity of pack reduces to the one of line 6 in Algorithm 1 times the number of initial configurations as all the other lines are constant in time. Each time a rectangle is packed, the number of new corners points in the border is 4 in the worst case (see cases (d) and (e) in Fig. 4). Assuming the container's area is sufficient to pack all the rectangles, the number of placements considered in the worst 
case for the second rectangle is 4,8 for the third one, 12 for the fourth one, $\ldots$ and $4(n-1)$ for the last rectangle. This sums up to $4(1+2+\ldots+(n-1))=4 \cdot \frac{n(n-1)}{2}$ which is $O\left(n^{2}\right)$. Therefore, the complexity of pack is $O\left(k n^{2}\right)$.

\subsection{An illustrative example}

We illustrate the procedure pack on the instance rect1 proposed by López and Beasley (2018b) which consists in packing 10 rectangles into a circular container of radius 3.62 without rotation. Details of the instance together with three lists of rectangles $l^{1}, l^{2}$ and $l^{3}$ which are given as inputs are presented in the upper-left part of Figure 5. The list $l^{1}$ (resp. $l^{2}$ ) is sorted in the decreasing (resp. increasing) order of the rectangles' areas. The list $l^{3}$ is obtained by means of a more sophisticated search described in Section 3 In Figure 5, the parts (i), (ii) and (iii) illustrate the packings returned by the pack procedure when applied on the lists $l^{1}, l^{2}$ and $l^{3}$ respectively. The last part presents the results for the various packings. For a given list, the procedure pack builds a packing for every possible initial configuration then returns the best packing obtained (see Section 2.3). The best initial configuration for $l^{1}$ turns to be $\mathrm{R}$ implying that rectangle 10 is initially packed so that $V_{10}^{1}$ and $V_{10}^{4}$ lie on the circle. The initial border is $\left(V_{10}^{1}, V_{10}^{2}, V_{10}^{3}, V_{10}^{4}\right)$. The next five rectangles in the list which are $9,8,7,6$ and 5 can not be packed by any predefined move. The best placement for rectangle 4 with regard to the border is to place this rectangle on the left of rectangle 10 according to the move (e) (see Section 2.4). Then, rectangle 3 is placed under rectangle 4 by the move (a). No more rectangle can be packed in this case. The resulting border is $\left(V_{10}^{1}, V_{10}^{2}, V_{4}^{1}, V_{4}^{2}, V_{4}^{3}, V_{3}^{2}, V_{3}^{3}, V_{3}^{4}, V_{10}^{3}, V_{10}^{4}\right)$. The best initial configuration for $l^{2}$ is $\mathrm{C}$ showing that rectangle 1 is packed at the centre. Rectangle 2 which is the next in the list is packed on rectangle 1 by the move (c). Then, rectangle 3 is packed under rectangle 2 by the move (a). The closest placement to the centre for rectangle 4 lies on the right of rectangle 1 by means of the move (c). No feasible placement is found for rectangle 5 but rectangle 6 can be packed under rectangle 1 by a little shift as allowed by the move (e). No more rectangles in $l^{2}$ can be packed. As for the list $l^{1}$, the best initial configuration for $l^{3}$ is $\mathrm{R}$ implying that rectangle 7 is packed on the right of the circle. The next rectangles $6,4,2,3$ and 1 are packed according to the moves (c), (a), (e), (a) and (a) respectively. No more rectangle can be packed by the predefined moves. In part (iv), when comparing the lists $l^{1}$ and $l^{2}$, the packing produced with $l^{1}$ covers a larger area than the one of $l^{2}$, whereas the packing generated with $l^{2}$ contains a larger number of items than the one of $l^{1}$. However, the list $l^{3}$ which is neither sorted in the decreasing nor the increasing order of rectangles' areas produces the packing with both the largest area and number of items packed.

As presented in this example, the quality of the packing obtained depends highly on the list of rectangles given as input to pack, a fortiori, on the first two rectangles in the list. 


\section{Instance and lists}

$n=10, R=3.62$,

$l^{1}=(10,9,8,7,6,5,4,3,2,1)$,

$l^{2}=(1,2,3,4,5,6,7,8,9,10)$,

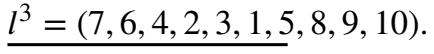

\begin{tabular}{ccc}
\hline$i$ & $L_{i}$ & $W_{i}$ \\
\hline 1 & 1.10 & 1.61 \\
2 & 2.20 & 1.08 \\
3 & 1.68 & 1.46 \\
4 & 1.82 & 2.61 \\
5 & 2.70 & 2.57 \\
6 & 3.21 & 2.21 \\
7 & 2.99 & 3.51 \\
8 & 3.68 & 3.42 \\
9 & 4.62 & 3.36 \\
10 & 3.79 & 4.79
\end{tabular}

(ii)

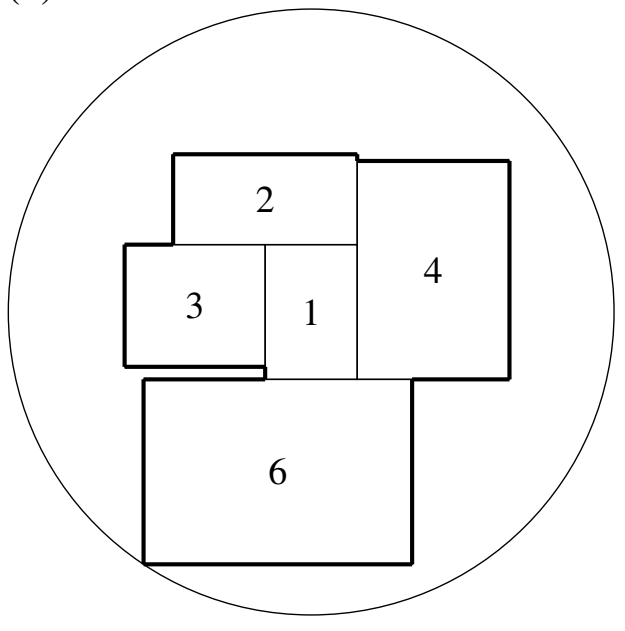

(i)

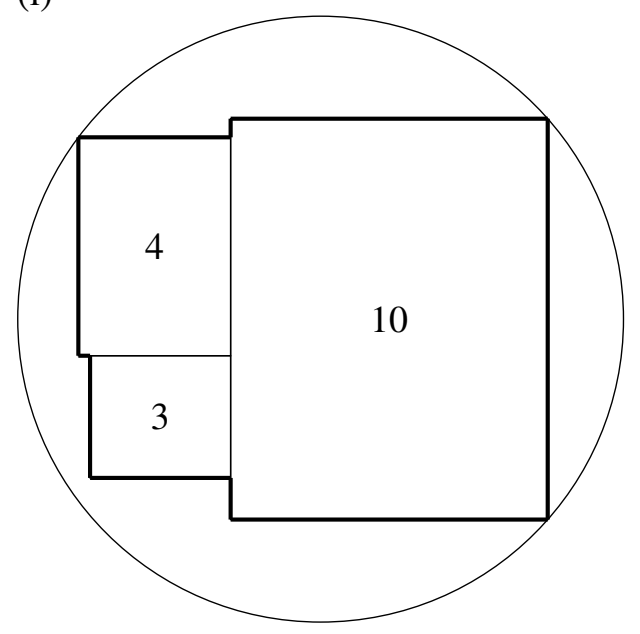

(iii)

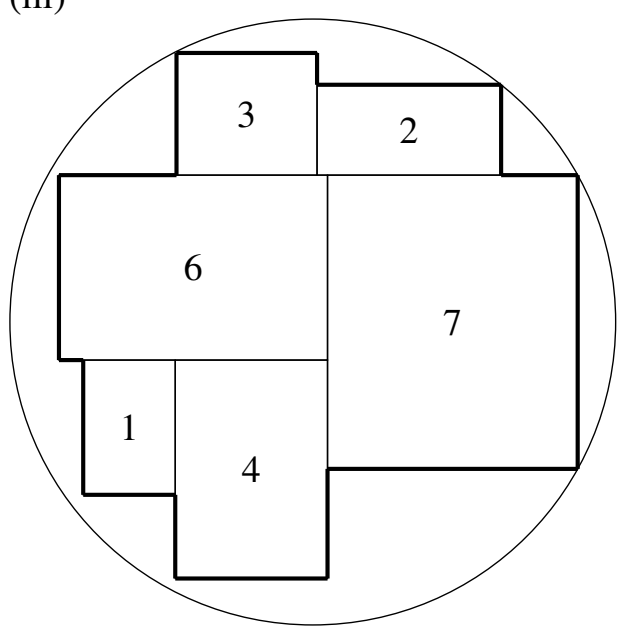

\begin{tabular}{cccc} 
(iv) & & & \\
\hline$l^{i}$ & Order & Packed area & Number of items \\
\hline$l^{1}$ & $\searrow$ & 25.3571 & 3 \\
$l^{2}$ & $\nearrow$ & 18.4441 & 5 \\
$l^{3}$ & - & 28.9390 & 6 \\
\hline
\end{tabular}

Fig. 5. Illustration of pack on the instance rect1 of López and Beasley (2018b) without rotation.

\subsection{Building the border of an already known packing}

In this section, we present algorithm 2 which builds and returns the border surrounding a connected packing $p$ given as input. During the process, the algorithm maintains a set $S$ of rectangles which are packed in $p$ but not surrounded by the border $b(p)$ yet. Initially, $b(p)$ receives the corners of a rectangle $u_{1}$ which is then removed from $S$. Then, while $S$ is not empty, the algorithm examines every edge $[A, B]$ forming $b(p)$ in order to find if there is a rectangle $\bar{u}=(C, D, E, F)$ with a side $[C, D]$ contiguous to $[A, B]$. If the rectangle $\bar{u}$ exists, its corners are integrated into $b(p)$ without forming duplicated nodes and $\bar{u}$ is removed from $S$ (see Figure 6). The algorithm terminates when $S$ is empty. 


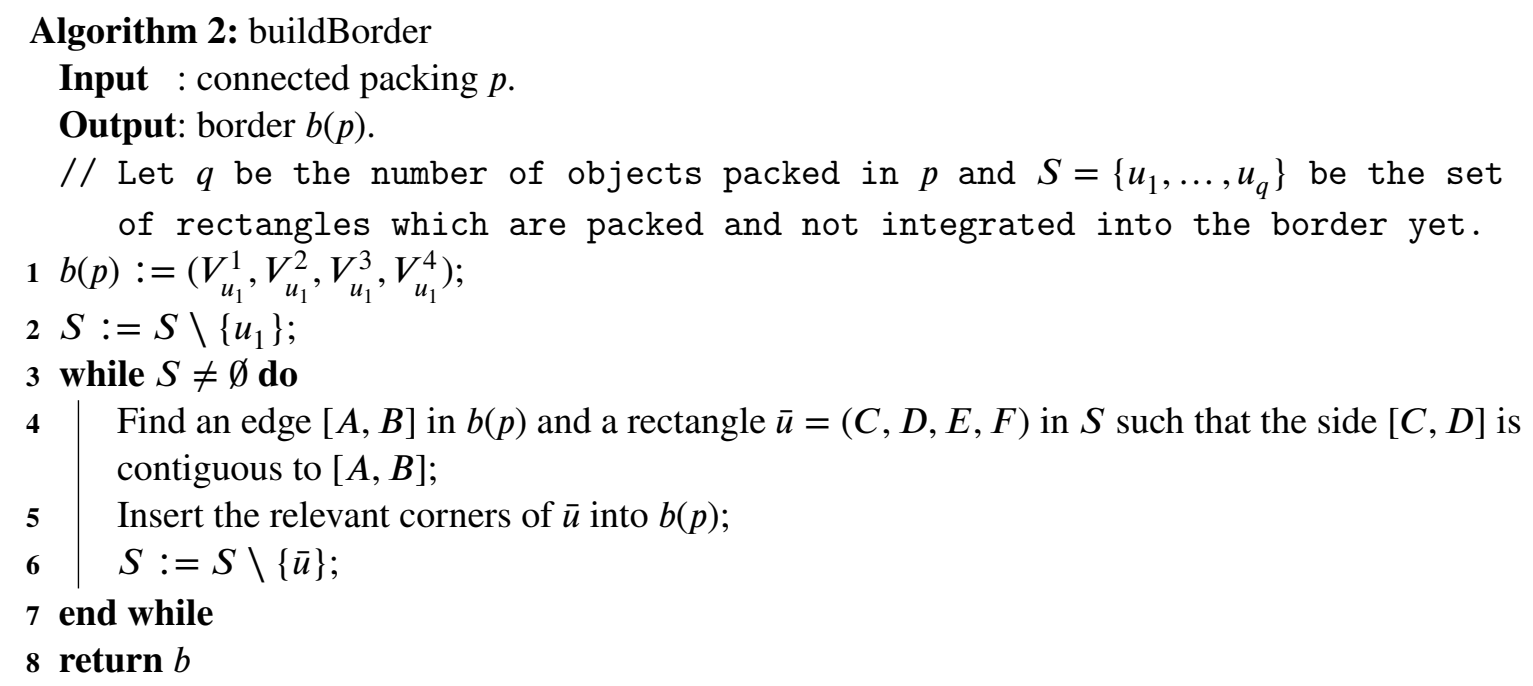

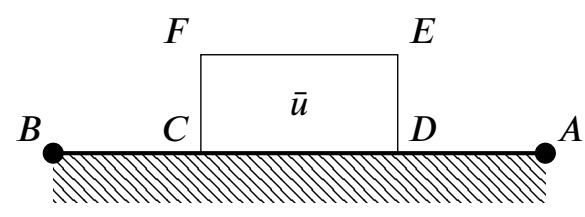

(a)

Fig. 6. Inserting the corners of rectangle $\bar{u}$ into the border.

Theorem 2. Let $p$ be a connected packing containing q rectangles then Algorithm 2 finds the border $b(p)$ surrounding $p$ in $O\left(q^{3}\right)$.

Proof. Given the connectedness of the packing $p$, every rectangle which is packed but not integrated yet into the border $b(p)$ will be reached and surrounded by the latter in the algorithm by means of the operations at lines 4 and 5 As all rectangles are integrated into $b(p)$ and every integrated rectangle is removed from $S$ (see line 6, the set $S$ becomes empty and the algorithm converges.

The time complexity of the algorithm equals the complexity of the while loop. The worst case occurs when the last edge in $b(p)$ is contiguous to the last rectangle examined in $S$ and when four new corners are inserted at every iteration. Depending on the positions of $A$ and $B$, we can determine in constant time whether the edge $[A, B]$ is a right, top, left or bottom edge of $b(p)$. Furthermore, depending on the orientation of $[A, B]$, we can limit the search for a contiguous rectangle's side $[C, D]$ to those which are confronting $[A, B]$. For instance, if $[A, B]$ is a right edge, we are looking for a left rectangle's side $[C, D]$ contiguous to $[A, B]$ and so on. As testing whether two segments are contiguous is constant in time, the number of operations performed at line 4 is $4 k(q-k)$ where $k$ is the number of rectangles already integrated into $b(p)$. The insertion and the removal at lines 5 -6 are constant in time if $b(p)$ and $S$ are implemented as chained lists. As a consequence, the complexity of the algorithm which equals the while loop's complexity corresponds to the number of pairs (edge $[A, B]$, rectangle's side $[C, D]$ ) 
examined during the whole process which is given in the worst case by:

$$
4(q-1)+8(q-2)+\ldots+4(q-1)=\sum_{k=1}^{q-1} 4 k(q-k)=\frac{2}{3} q\left(q^{2}-1\right)=O\left(q^{3}\right)
$$

It is worth mentioning that Algorithm 2 can be easily adapted to determine the decomposition of a packing into connected components. Indeed, starting from a rectangle $u$ given as an input, the algorithm determines the connected partial packing to which $u$ belongs. If all the rectangles have been inserted into $b(p)$ then the packing is connected. Otherwise, the algorithm restarts from another rectangle which does not belong to the connected component of $u$ to determine its connected component and so on.

\section{Integration of pack into two metaheuristics}

The application of pack on one single list is a simple constructive heuristic which may obviously lead to poor local optima. One way forward to increase the chances in providing a globally optimal packing is then to incorporate pack into a metaheuristic. As an illustration only, Hifi and Yousef (2019) provides a successful integration of a constructive heuristic into an effective metaheuristic scheme for the packing of spheres into a cuboid. In this section, we first review briefly some applications of two powerful metaheuristics, namely, variable neighbourhood search (VNS) and simulated annealing (SA), to packing problems. We then present two integrations of the procedure pack to these two metaheuristics. Moreover, based on Algorithm 2, we propose two accelerated variants of these methods. The purpose of these integrations is to visit a broad set of lists in order to maximise the chances to reach a global optimum. For completeness, we terminate this section by a discussion on the nature of our methodology and how it is situated in the field of metaheuristics.

\subsection{Applications of VNS and SA to packing problems in the literature}

VNS is a metaheuristic developed by Mladenović and Hansen (1997) which consists in applying systematic change of neighbourhood within a local search algorithm. VNS (and its variants) have been applied successfully to several packing problems. For instance, Mladenović et al. (2005; 2007) applied reformulation descent and FSS to the packing of equal circles into a unit circle respectively, enabling a significant speed-up over existing methods and packing up to 100 circles. López and Beasley (2011) proposed a competitive FSS approach for the packing of equal circles into a variety of containers and packing up to 500 circles. M'Hallah and Alkandari(2012) adopted a VNS for the packing of unit spheres into the smallest cube where the local search is performed by a non-linear programming solver. Later, M'Hallah et al. (2013) applied a similar approach to the packing of unit spheres into the smallest sphere and pro- 
duced 29 new upper bounds out of 48 benchmark instances. López and Beasley (2013) extended their earlier work by tackling the problem of packing unequal circles having a variable size into a container of fixed size having various shapes. They proposed a FSS method coupled with a perturbation phase. Instances involving up to 35 objects where considered. In a subsequent study, López and Beasley (2016) addressed the problem of packing a subset of unequal circles into a circular container of fixed size where the choice of the subset of circles to pack is also a decision variable. They proposed a FSS approach that they test on instances they generated and which involve up to 40 circles. Zeng et al. (2016) combined tabu search and variable neighbourhood descent to tackle the packing of unequal circles into a circular container with the aim to minimise the radius. In their following study, Zeng et al. (2018) proposed a similar metaheuristic for the packing of unequal circles into a square of minimum side length.

Simulated annealing (SA) is a metaheuristic introduced by Kirkpatrick et al. (1983). It results from an analogy between optimisation and the annealing process found in the steel industry. SA has been applied successfully by several authors to the packing of rectangles into a larger containing rectangle (Dowsland, 1993, Leung et al., 2003; Soke and Bingul, 2006, Leung et al., 2011). Gomes and Oliveira (2006) considered the more general problem of packing irregular shapes into a rectangular container which they successfully addressed by hybridising SA and linear programming. Martins and Tsuzuki (2010) efficiently applied SA to another general problem which consists in packing irregular polygons into a container while allowing arbitrary rotations. Hinostroza et al. (2013) developed a SA for a packing problem similar to the one we consider though no rotation is allowed in their algorithm. This latter relies heavily on an ordering heuristic which packs rectangles in a specified order. The dataset on which they assessed the performance of their method has not been made public. In this section, we present an alternative simulated annealing which integrates the procedure pack.

For further reading, Salhi (2017) provides an informative description of metaheuristics in general including VNS and SA. Very recently, Hansen et al. (2019) produces an updated chapter that presents the basic schemes and extensions as well as recent developments of VNS.

\subsection{Integration of pack into a VNS scheme}

Algorithm 3 is a variable neighbourhood search. It requires initially three parameters $k_{\max }, i t_{\max }$ and $l_{0}$ which represent the maximum number of neighbourhood structures, the maximum number of nonimproving iterations allowed and an initial ordered list of the $n$ rectangles respectively. It returns the best packing $p_{\text {best }}$ found so far. As described above, our VNS explores the lists search space. The neighbourhood structure used is defined by $N_{k}(l)$ which constitutes the set of lists obtained by swapping $k$ pairs of rectangles indices in the list $l$ with $1 \leq k \leq k_{\max }$. These neighbourhoods are explored by the procedure $\operatorname{shake}\left(l_{\text {best }}, k\right)$ which returns a list obtained by swapping randomly $k$ pairs of indices in the list $l_{\text {best }}$ (see 
line 7). In order to convert a list $l$ into a packing, the algorithm relies on the decoder pack $(l)$ which constitutes a key component of our methodology (see line 8). Overall, our algorithm consists of two nested loops. The inner one explores the lists and packings search spaces by means of the two procedures shake and pack (see lines 5. 18). At this step, the list $l_{\text {best }}$ which permitted to find the best packing $p_{\text {best }}$ so far is shaken to generate a new list $l$ using the shake procedure. A new packing $p$ is built from $l$ using the procedure pack. If the value associated with $p$ is better than the best value found so far, the search is recentred on $l$ by reinitialising $k$ and the outer loop iterator (see lines 9] 13 , otherwise, the search is enlarged around $l_{\text {best }}$ (see line 15). The outer loop (lines 3 19) controls the overall process by limiting the number of non-improving iterations to $i t_{\max }$.

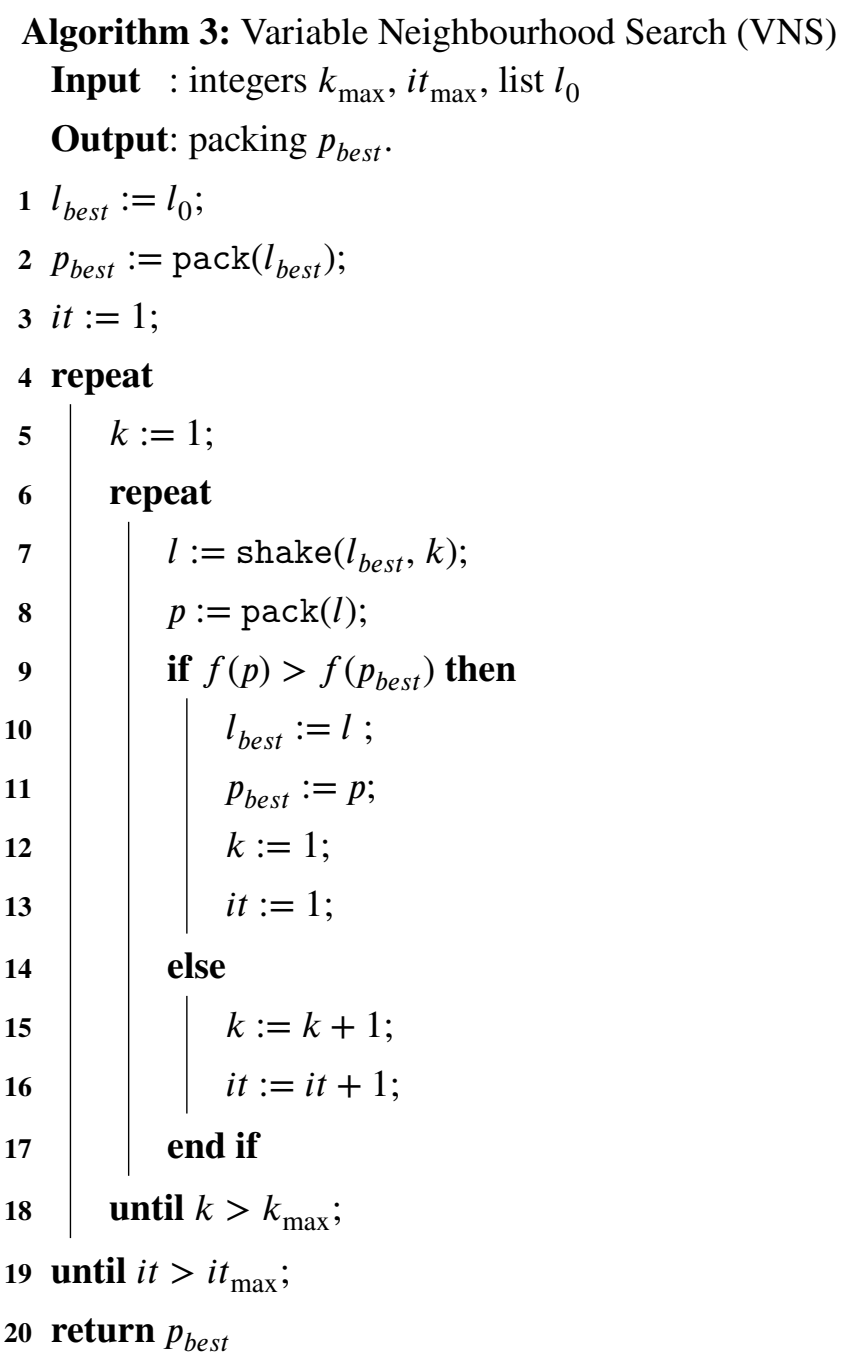

\subsection{Integration of pack into a SA scheme}

Algorithm 4 is a simulated annealing metaheuristic that incorporates the procedure pack. It receives as input $T_{0}, T_{f}, \alpha, N_{t}, k, i t_{\max }$ and $l_{0}$ which are the initial and the final temperatures, the cooling factor, the number of iterations spent at every temperature, the number of rectangles to swap in the shake procedure, the maximum number of non-improving iterations allowed and the initial list respectively. It returns the 
best packing found by the algorithm. Initially, the temperature $T$ is set to $T_{0}$ and the incumbent packing $p$, which is considered as the best packing at this step, is generated from $l_{0}$ using pack. At every iteration of the main loop, a neighbouring list $l^{\prime}$ is generated by swapping randomly $k$ rectangles in the incumbent list $l$. The resulting list is transformed into a candidate packing $p^{\prime}$ by using pack and the temperature is updated by calcTemp $(i, T)$. This function performs the classical geometric reduction $T:=\alpha T$ every $N_{t}$ iterations. If the value of the candidate packing is better than the incumbent one (see line 9), the latter is replaced by the former and a new test is made to check if the candidate packing improves the best packing found so far in which case the best list and packing are updated. In case the value of the candidate packing is not better than the incumbent one, it is accepted only if it satisfies the well known Boltzmann acceptance rule (see line 14). The main loop is performed until a stopping criterion is reached. In our case, this refers to the maximum number of iterations which is $N_{t}\left\lceil\frac{\ln T_{f}-\ln T_{0}}{\ln \alpha}\right\rceil$ or $i t_{\max }$ iterations without any improvement made to the objective function whichever comes first.

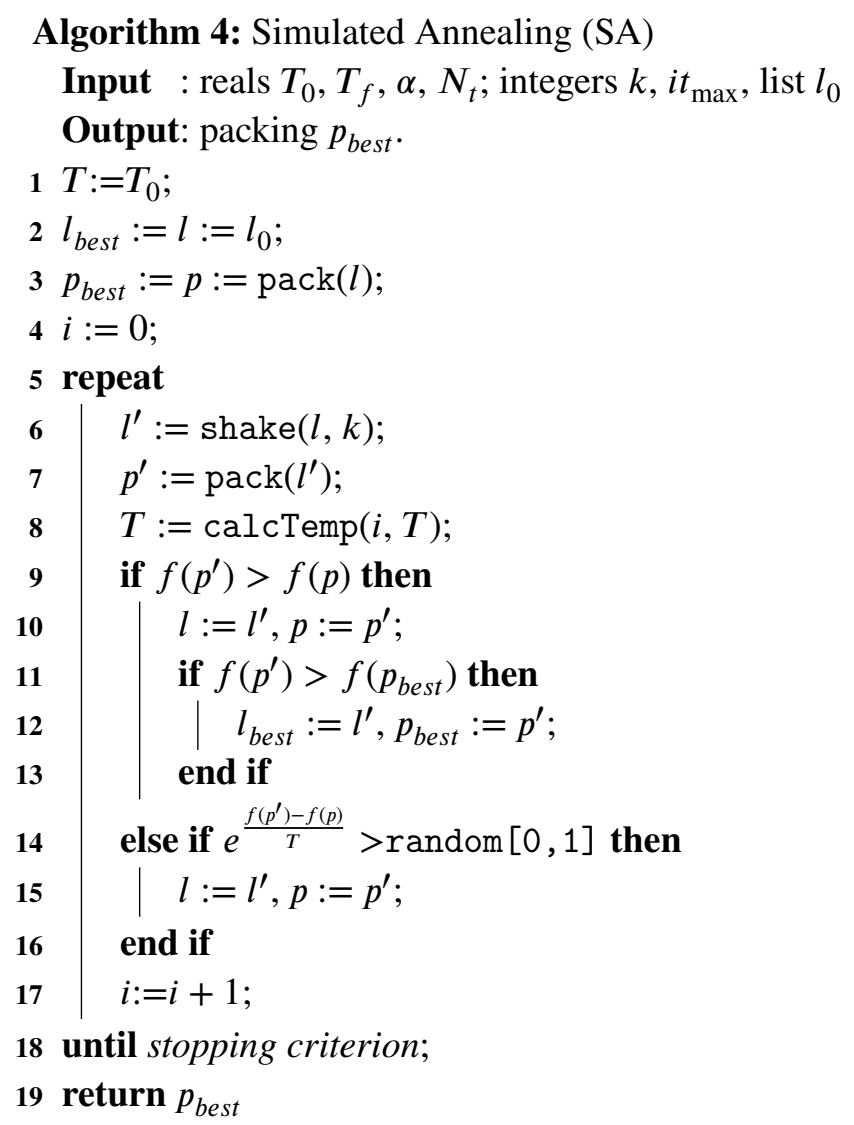

\subsection{Speed-up mechanisms}

In heuristic search design, the number of iterations is usually very large and the solution configurations from one iteration to the next have a lot of in common. It is therefore crucial to avoid recomputing already processed parts of earlier configurations. Exploiting this aspect can reduce the computational 
time considerably turning the search into a much faster one. Some of these powerful techniques are discussed in Salhi (2017, Chapter 6).

Let $i$ be the lowest index such that $l_{i}$ is relocated by the shake procedure at line 7 in VNS and line 6 in SA. When the pack procedure is called at line 8 in VNS, if the initial configuration of the packing $p_{\text {best }}$ is preserved then the placement of the rectangles $l_{1}$ to $l_{i-1}$ in $p$ is the same as the one in $p_{\text {best }}$. Exploiting this idea for VNS and SA can considerably speed-up the search though the final solution quality may be deteriorated as a variation of the initial configuration is less likely to occur.

a) Case of VNS - We propose a variant of VNS called xVNS where the lines 7 -8 are replaced by the two following lines:

$$
\begin{aligned}
& <l, i>:=\operatorname{xShake}\left(l_{\text {best }}, k\right) \\
& p:=\operatorname{xPack}\left(l, i, p_{\text {best }}\right) ;
\end{aligned}
$$

The procedure $\mathrm{xShake}\left(l_{\text {best }}, k\right)$ returns a copy $l$ of $l_{\text {best }}$ where $k$ pairs of elements have been swapped randomly, $i$ being the smallest index of an element relocated in $l$. This function returns both $l$ and $i$. The prcedure $\mathrm{xPack}$ is an extension of the procedure pack which copies from $p_{\text {best }}$ into $p$ the placements of the packed rectangles in the list $l_{1} \ldots l_{i-1}$, builds the border surrounding these rectangles by means of Algorithm 2 then pack the remaining rectangles in $l_{i} \ldots l_{n}$ as performed in the while-loop (lines 5 to 11 ) of the procedure pack.

b) Case of SA - Similarly to case (a), we propose a variant of SA called xSA where the lines 6 67 are replaced by the two following lines:

$$
\begin{aligned}
& <l^{\prime}, i>:=\operatorname{xShake}(l, k) \\
& p^{\prime}:=\operatorname{xPack}\left(l^{\prime}, i, p\right)
\end{aligned}
$$

\subsection{Some observations}

Among the existing variants of VNS (see Hansen et al. (2010)), Algorithm 3 is closest to the Reduced VNS (RVNS) as it consists of a shaking procedure but no local search. From this point of view, the search space explored in Algorithm 3 is the one of permutations whereas the pack procedure plays the role of an evaluation procedure used to assess the ability of a given permutation in producing a good packing. The composite function $f(\operatorname{pack}(l))$ used at line 9 of Algorithm 3 is then a non-constant time cost function (see Theorem 1) used during the neighbourhood change step of RVNS.

The idea of exploring an alternative search space instead of exploring directly the solution search space is present in the literature. By definition, evolutionary algorithms make intensive use of encoding procedure to convert solutions (phenotypes) into representations (genotypes) (Talbi, 2009, Section 1.4.1). 
In the context of vehicle routing, Prins et al. (2014) reviews more than 70 references related to the class of so-called "order-first split-second" methods. These methods oscillate between two search spaces by means of a decoding and encoding procedures called Split and Split ${ }^{-1}$ respectively. The encoding procedure permits to convert a routing into a permutation whereas the decoding procedure determines a routing by optimally partitioning a permutation.

Our methodology follows a simpler architecture as it uses only a perturbation procedure, namely shake, to explore the permutation search space, and a decoding procedure, namely pack, used to convert a permutation into a packing. The resulting algorithms (VNS and SA) are still trajectorial metaheuristics with the nuance that the path they describe is inscribed in the alternative search space; not in the solution search space. The search is thus focused on permutations and, in some sense, the packings visited during the search are only "evaluations" of the permutations encountered in the alternative search space. Based on the quality of the packings resulting from the visited permutations, the search is guided towards the "best" permutation; the one that results in a packing of best known quality when it is given as input to pack.

We demonstrate in the next section that our simplistic approach reveals highly effective and efficient when tested on benchmark instances.

\section{Computational experiments}

The experiments are conducted on an Intel Core i3-2330M @ $2.20 \mathrm{GHz}$ with 3.9 Gbytes RAM running Linux and the algorithms are coded in Java. They consist of three main parts. First, we assess the performance of both the SA and the VNS algorithms with the algorithm of López and Beasley (2018b) (denoted FSS) on the dataset they proposed and which is available from López and Beasley (2018a). To the best of our knowledge, this is the only publicly available dataset for this packing problem. Second, we assess the statistical significance of the results obtained in the first part using the Friedman test. Finally, we generate larger instances to be used for a comparative study between SA, VNS and their respective accelerated variants $\mathrm{xSA}$ and $\mathrm{xVNS}$.

\subsection{Datasets and parameter calibration}

\subsubsection{Existing dataset}

The dataset proposed by López and Beasley (2018b) consists of instances involving $n=10,20,30$ rectangles or squares with randomly generated dimensions. For each instance, three different container radii are considered. They are calculated based on a formula involving a fraction $\rho$ of the total area of the objects to pack. For all instances, both the maximisation of the total area packed and the maximisation 
of the number of objects packed are considered. For instances involving rectangles, two supplementary cases are considered which are the possibility to rotate or not the rectangles. In total, this dataset consists of 54 instances.

\subsubsection{Newly constructed large dataset}

In addition and following the generating scheme proposed López and Beasley (2018b), we generated a new set of larger instances involving $n=100,150,200$ rectangles/squares. Their dimensions were pseudo-randomly generated (to two decimal places) in the interval $[1,5]$. For each instance, three container radii $R$ are set so that the container's area represents $\frac{1}{3}, \frac{1}{2}$ and $\frac{2}{3}$ of the total area of the $n$ objects to pack. This new dataset alongside all the new best known solutions obtained in this paper are made publicly available at the CLHO (2019) website. These informations can be useful for benchmarking purposes.

\subsubsection{Parameter calibration for VNS \& SA}

To calibrate our metaheuristics, we carried out experiments on a pseudo-randomly generated instance which consists of 100 rectangles. For VNS, the three parameters $k_{\max }, i t_{\max }$ and $l_{0}$ need to be set, while, for SA there are seven parameters, namely, $T_{0}, T_{f}, \alpha, N_{t}, k, i t_{\max }$ and $l_{0}$. Preliminary results showed clearly that when the objective is to maximise the area (resp. maximise the number of objects) packed, $l_{0}$ should be sorted in decreasing (resp. increasing) order of the object areas.

In the case of VNS, three values were tested for $k\left(3,5\right.$ and 7) and two for $i t_{\max }\left(5 \times 10^{2}\right.$ and $\left.10^{3}\right)$. Five runs were performed for every combination. The best compromise in terms of solution quality and computation time turned out to be $k=3$ and $i t_{\max }=5 \times 10^{2}$.

In the case of SA, the number of possibilities was much larger so we limited the number of values to two for all parameters except the number of runs to three per combination. The tested values were as follows: $T_{0}(10,20), T_{f}(0,01,0.1), \alpha(0.95,0.98), N_{t}(5,10), k(3,7)$ and $i t_{\max }\left(10^{3}\right.$ and $\left.10^{4}\right)$. The selected combination uses $T_{0}=10, T_{f}=0.1, \alpha=0.98, N_{t}=5, k=3$ and $i t_{\max }=10^{4}$.

Complementary data relevant to this preliminary experimentation are presented in Appendix A

\subsection{Results on the López and Beasley's dataset (2018a)}

We first present the overall results and then analyse their statistical significance.

\subsubsection{Overall results}

Tables 24 and Tables 5 -7 present the results for the cases where the objective is the maximisation of the number of objects packed and the maximisation of the area packed respectively. All Tables 2 , 7 follow 
the same structure. The first two columns identify the instance. The next two columns report the results (solution and computation time) presented by López and Beasley (2018b) for FSS. The columns labelled $\operatorname{Val}\left(l_{\text {inc }}\right)$ and $\operatorname{Val}\left(l_{d e c}\right)$ indicate the results obtained by applying pack on a list of the rectangles sorted in increasing and decreasing order of their areas respectively. Columns labelled SA and VNS present the results obtained by applying five runs of SA and VNS algorithms respectively. Both SA and VNS algorithms make an extensive use of the shake procedure which requires a pseudo-random generator when implemented. For every algorithm and for every instance, different runs are conducted in order to vary the initial random seed used in the search. This is performed to assess the robustness of SA and VNS. Columns labelled Best and Avg indicate the best solution value found and the average solution value over all the runs performed. Columns labelled T(s) correspond to the total time (in seconds) spent by the algorithms over all the runs performed. The computation time for a single run of the procedure pack is negligible. The last column (BKS) corresponds to the best known solution value including the ones found in this paper. Bold and underlined-bold values indicate a best solution value and a new best solution value respectively. The last rows provide average measures over the instances and the number of best values obtained. Average measures include the average difference calculated when the problem is to maximise the number of objects packed where the difference of a value Val is Diff = Best - Val, the average deviation calculated when the problem is to maximise the area packed where the deviation of a value Val is $\operatorname{Dev}(\%)=100 \times \frac{\text { Best-Val }}{\text { Best }}$, and the average computation time in seconds.

Table 2

Results in the case of maximising the number of rectangles packed without rotation

\begin{tabular}{|c|c|c|c|c|c|c|c|c|c|c|c|c|}
\hline \multirow[t]{2}{*}{$n$} & \multirow[t]{2}{*}{$\rho$} & \multicolumn{2}{|c|}{ FSS } & \multicolumn{2}{|c|}{ pack } & \multicolumn{3}{|c|}{ SA } & \multicolumn{3}{|c|}{ VNS } & \multirow[b]{2}{*}{ BKS } \\
\hline & & Best & $\mathrm{T}(\mathrm{s})$ & $\operatorname{Val}\left(l_{i n c}\right)$ & $\operatorname{Val}\left(l_{d e c}\right)$ & Best & Avg & $\mathrm{T}(\mathrm{s})$ & Best & Avg & $\mathrm{T}(\mathrm{s})$ & \\
\hline \multirow[t]{3}{*}{10} & \multirow{3}{*}{$\frac{1}{3}$} & 5 & 3058 & 4 & 2 & 5 & 5.00 & 1 & 5 & 4.40 & 1 & 5 \\
\hline & & 6 & 2862 & 5 & 4 & 6 & 6.00 & 2 & 6 & 6.00 & 1 & 6 \\
\hline & & 7 & 2966 & 6 & 6 & 7 & 7.00 & 2 & 7 & 7.00 & 1 & 7 \\
\hline \multirow[t]{3}{*}{20} & $\frac{1}{3}$ & 7 & 6278 & 6 & 5 & 7 & 7.00 & 5 & 7 & 7.00 & 2 & 7 \\
\hline & $\frac{3}{2}$ & 10 & 4530 & 8 & 7 & 10 & 10.00 & 5 & 10 & 10.00 & 2 & 10 \\
\hline & $\frac{2}{3}$ & 11 & 7311 & 11 & 9 & $\underline{13}$ & 13.00 & 6 & 12 & 12.00 & 2 & $\underline{13}$ \\
\hline \multirow[t]{3}{*}{30} & $\frac{1}{3}$ & 13 & 11514 & 13 & 7 & $\underline{14}$ & $\underline{14.00}$ & 10 & $\underline{14}$ & 13.80 & 7 & $\underline{14}$ \\
\hline & $\frac{1}{2}$ & 16 & 10029 & 15 & 10 & 18 & 18.00 & 11 & 17 & 17.00 & 5 & 18 \\
\hline & $\frac{2}{3}$ & 19 & 6966 & 17 & 15 & $\underline{21}$ & $\underline{21.00}$ & 13 & $\underline{21}$ & 20.20 & 8 & $\underline{21}$ \\
\hline \multirow{2}{*}{\multicolumn{2}{|c|}{$\begin{array}{c}\text { Avg. diff. or T(s) } \\
\text { \# Best }\end{array}$}} & 0.78 & 6168 & 1.78 & 4.00 & 0.00 & 0.00 & 6 & 0.22 & 0.40 & 3 & \\
\hline & & 5 & & 0 & 0 & 9 & 9 & & 7 & 4 & & \\
\hline
\end{tabular}

In terms of solution quality, when the objective is to maximise the number of objects (area resp.) packed, the procedure pack provides the best results when the list is sorted in increasing (decreasing resp.) order of the rectangles' areas. It is clear that one single run of pack is outperformed by SA and VNS. When the objective is to maximise the number of objects packed, SA dominates both VNS and FSS while remaining very stable (the average difference is less or equal to 0.09 ). VNS is superior to FSS 
Table 3

Results in the case of maximising the number of rectangles packed with rotation

\begin{tabular}{|c|c|c|c|c|c|c|c|c|c|c|c|c|}
\hline \multirow[t]{2}{*}{$n$} & \multirow[t]{2}{*}{$\rho$} & \multicolumn{2}{|c|}{ FSS } & \multicolumn{2}{|c|}{ pack } & \multicolumn{3}{|c|}{ SA } & \multicolumn{3}{|c|}{ VNS } & \multirow[b]{2}{*}{ BKS } \\
\hline & & Best & $\mathrm{T}(\mathrm{s})$ & $\operatorname{Val}\left(l_{i n c}\right)$ & $\operatorname{Val}\left(l_{d e c}\right)$ & Best & Avg & $\mathrm{T}(\mathrm{s})$ & Best & Avg & $\mathrm{T}(\mathrm{s})$ & \\
\hline \multirow[t]{3}{*}{10} & \multirow{3}{*}{$\begin{array}{l}\frac{1}{3} \\
\frac{1}{2} \\
\frac{2}{3}\end{array}$} & 5 & 9836 & 4 & 2 & 5 & 5.00 & 2 & 5 & 4.80 & 2 & 5 \\
\hline & & 6 & 10332 & 5 & 4 & 6 & 6.00 & 3 & 6 & 6.00 & 1 & 6 \\
\hline & & 7 & 12409 & 6 & 6 & 7 & 7.00 & 3 & 7 & 7.00 & 1 & 7 \\
\hline \multirow[t]{3}{*}{20} & $\frac{1}{3}$ & 8 & 22759 & 6 & 5 & 8 & 7.60 & 9 & 8 & 7.80 & 4 & 8 \\
\hline & $\frac{1}{2}$ & 10 & 30682 & 8 & 7 & 10 & 10.00 & 10 & 10 & 10.00 & 4 & 10 \\
\hline & $\frac{2}{3}$ & 12 & 30823 & 11 & 9 & $\underline{13}$ & $\underline{13.00}$ & 11 & 12 & 12.00 & 4 & $\underline{13}$ \\
\hline \multirow[t]{3}{*}{30} & $\frac{1}{3}$ & 14 & 49724 & 13 & 6 & 14 & 14.00 & 17 & 14 & 14.00 & 10 & 14 \\
\hline & $\frac{1}{2}$ & 17 & 45857 & 15 & 10 & $\underline{18}$ & $\underline{18.00}$ & 19 & 18 & 17.20 & 11 & $\underline{18}$ \\
\hline & $\frac{2}{3}$ & 20 & 57427 & 18 & 15 & $\underline{21}$ & $\underline{21.00}$ & 20 & 20 & 20.00 & 10 & $\underline{21}$ \\
\hline \multirow{2}{*}{\multicolumn{2}{|c|}{$\begin{array}{c}\text { Avg. diff. or T(s) } \\
\text { \# Best }\end{array}$}} & 0.33 & 29983 & 1.78 & 4.22 & 0.00 & 0.04 & 10 & 0.22 & 0.36 & 5 & \\
\hline & & 6 & & 0 & 0 & 9 & 8 & & 7 & 4 & & \\
\hline
\end{tabular}

Table 4

Results in the case of maximising the number of squares packed

\begin{tabular}{|c|c|c|c|c|c|c|c|c|c|c|c|c|}
\hline \multirow[t]{2}{*}{$n$} & \multirow[t]{2}{*}{$\rho$} & \multicolumn{2}{|c|}{ FSS } & \multicolumn{2}{|c|}{ pack } & \multicolumn{3}{|c|}{ SA } & \multicolumn{3}{|c|}{ VNS } & \multirow[b]{2}{*}{ BKS } \\
\hline & & Best & $\mathrm{T}(\mathrm{s})$ & $\operatorname{Val}\left(l_{i n c}\right)$ & $\operatorname{Val}\left(l_{d e c}\right)$ & Best & Avg & $\mathrm{T}(\mathrm{s})$ & Best & Avg & $\mathrm{T}(\mathrm{s})$ & \\
\hline \multirow[t]{3}{*}{10} & \multirow{3}{*}{$\begin{array}{l}\frac{1}{3} \\
\frac{1}{2} \\
\frac{2}{3}\end{array}$} & 4 & 1123 & 3 & 1 & 4 & 4.00 & 1 & 4 & 4.00 & 1 & 4 \\
\hline & & 5 & 2761 & 4 & 4 & 5 & 5.00 & 1 & 5 & 5.00 & 1 & 5 \\
\hline & & 6 & 2275 & 5 & 4 & 6 & 6.00 & 2 & 6 & 6.00 & 1 & 6 \\
\hline \multirow[t]{3}{*}{20} & $\frac{1}{3}$ & 11 & 5450 & 9 & 10 & 11 & 11.00 & 5 & 10 & 10.00 & 2 & 11 \\
\hline & $\frac{1}{2}$ & 12 & 6465 & 11 & 11 & $\underline{13}$ & 12.20 & 5 & 12 & 11.80 & 3 & $\underline{13}$ \\
\hline & $\frac{2}{3}$ & 14 & 6995 & 12 & 13 & 14 & 14.00 & 6 & 14 & 13.20 & 3 & 14 \\
\hline \multirow[t]{3}{*}{30} & $\frac{1}{3}$ & 16 & 13552 & 14 & 13 & 16 & 16.00 & 10 & 15 & 15.00 & 6 & 16 \\
\hline & $\frac{1}{2}$ & 20 & 13457 & 18 & 13 & 20 & 20.00 & 12 & 20 & 19.60 & 8 & 20 \\
\hline & $\frac{2}{3}$ & 23 & 10427 & 21 & 17 & 23 & 23.00 & 13 & 22 & 22.00 & 7 & 23 \\
\hline \multirow{2}{*}{\multicolumn{2}{|c|}{$\begin{array}{c}\text { Avg. diff. or T(s) } \\
\text { \# Best }\end{array}$}} & 0.11 & 6945 & 1.67 & 2.89 & 0.00 & 0.09 & 6 & 0.44 & 0.60 & 3 & \\
\hline & & 8 & & 0 & 0 & 9 & 8 & & 5 & 3 & & \\
\hline
\end{tabular}

when dealing with rectangle packing but FSS is able to provide better solutions when the problem is to pack squares. When the objective is to maximise the area packed, both SA and VNS dominate FSS but SA and VNS are not dominating each other. In total, 32 new best solutions out of 54 are found.

In terms of computational effort, SA and VNS are clearly faster than FSS though the computer used with FSS, the Intel Core i5-2400S @ $2.50 \mathrm{GHz}$, is ranked better than our computer in terms of CPU performance (PassMark, 2020). However, it is important to mention that FSS is a formulation search space approach which relies heavily on a third-party solver which slows the whole process.

We can conclude that, for this implementation of FSS and on this particular dataset, both VNS and SA are able, in the majority of cases, to find better solutions than FSS while being relatively much faster. There is however no obvious conclusion that can be made between SA and VNS. 
Table 5

Results in the case of maximising the area of rectangles packed without rotation

\begin{tabular}{|c|c|c|c|c|c|c|c|c|c|c|c|c|}
\hline \multirow[t]{2}{*}{$n$} & \multirow[t]{2}{*}{$\rho$} & \multicolumn{2}{|c|}{ FSS } & \multicolumn{2}{|c|}{ pack } & \multicolumn{3}{|c|}{ SA } & \multicolumn{3}{|c|}{ VNS } & \multirow[b]{2}{*}{ BKS } \\
\hline & & Best & $\mathrm{T}(\mathrm{s})$ & $\operatorname{Val}\left(l_{i n c}\right)$ & $\operatorname{Val}\left(l_{d e c}\right)$ & Best & Avg & $\mathrm{T}(\mathrm{s})$ & Best & Avg & $\mathrm{T}(\mathrm{s})$ & \\
\hline \multirow[t]{3}{*}{10} & $\frac{1}{3}$ & 18.4441 & 3292 & 11.35 & 17.8992 & 18.4441 & 18.4441 & 5 & 18.4441 & 18.3351 & 1 & 18.4441 \\
\hline & $\frac{3}{2}$ & 28.9390 & 2992 & 18.4441 & 25.3571 & 28.9390 & 28.9390 & 2 & 28.9390 & 28.9390 & 1 & 28.9390 \\
\hline & $\frac{2}{3}$ & 37.6878 & 4754 & 25.3831 & 36.5982 & $\underline{39.4588}$ & 38.9214 & 2 & 38.7870 & 38.7870 & 1 & $\underline{39.4588}$ \\
\hline \multirow[t]{3}{*}{20} & $\frac{3}{3}$ & 43.3885 & 7227 & 31.5072 & 41.7542 & $\overline{45.1727}$ & 45.0616 & 5 & 45.1567 & 44.7210 & 2 & 45.1727 \\
\hline & $\frac{1}{2}$ & 63.1643 & 9791 & 42.7353 & 64.5214 & 69.9263 & 68.5124 & 6 & 68.8314 & 67.4388 & 3 & 69.9263 \\
\hline & $\frac{2}{3}$ & 84.4446 & 10601 & 70.1397 & 87.5713 & $\underline{93.0556}$ & 91.3516 & 6 & 91.6368 & 90.6159 & 3 & $\underline{93.0556}$ \\
\hline \multirow[t]{3}{*}{30} & $\underline{1}$ & 60.3570 & 14011 & 48.1206 & 59.8421 & 65.2856 & 64.5676 & 10 & 64.4689 & 63.7051 & 5 & 65.2856 \\
\hline & $\frac{3}{2}$ & 85.2113 & 19786 & 63.6659 & 95.3117 & $\overline{100.1839}$ & 98.4151 & 12 & $\underline{102.1196}$ & 99.3385 & 8 & $1 \overline{102.1196}$ \\
\hline & $\frac{2}{3}$ & 103.4802 & 19470 & 80.064 & 131.1013 & 135.9217 & 134.3052 & 13 & $\underline{137.4149}$ & 135.2876 & 7 & 137.4149 \\
\hline \multirow{2}{*}{\multicolumn{2}{|c|}{$\begin{array}{c}\text { Avg. dev (\%) } \\
\text { or T(s) } \\
\text { \# Best }\end{array}$}} & 8.46 & 10214 & 34.43 & 7.04 & 0.33 & 1.38 & 7 & 0.68 & 1.80 & 3 & \\
\hline & & 2 & & 0 & 0 & 7 & 2 & & 4 & 1 & & \\
\hline
\end{tabular}

Table 6

Results in the case of maximising the area of rectangles packed with rotation

\begin{tabular}{|c|c|c|c|c|c|c|c|c|c|c|c|c|}
\hline \multirow[t]{2}{*}{$n$} & \multirow[t]{2}{*}{$\rho$} & \multicolumn{2}{|c|}{ FSS } & \multicolumn{2}{|c|}{ pack } & \multicolumn{3}{|c|}{ SA } & \multicolumn{3}{|c|}{ VNS } & \multirow[b]{2}{*}{ BKS } \\
\hline & & Best & $\mathrm{T}(\mathrm{s})$ & $\operatorname{Val}\left(l_{\text {inc }}\right)$ & $\operatorname{Val}\left(l_{d e c}\right)$ & Best & Avg & $\mathrm{T}(\mathrm{s})$ & Best & Avg & $\mathrm{T}(\mathrm{s})$ & \\
\hline \multirow[t]{3}{*}{10} & $\frac{1}{3}$ & 19.6702 & 8771 & 11.35 & 17.8992 & 19.6702 & 19.6702 & 3 & 19.6702 & 19.2633 & 1 & 19.6702 \\
\hline & $\frac{1}{2}$ & 29.5041 & 16093 & 18.4441 & 27.1281 & $\underline{30.8746}$ & 29.7782 & 3 & $\underline{30.8746}$ & 29.7208 & 1 & $\underline{30.8746}$ \\
\hline & $\frac{2}{3}$ & 37.9687 & 15526 & 25.3831 & 39.999 & 41.1612 & 40.8778 & 3 & 40.9063 & 40.3619 & 1 & 41.1612 \\
\hline \multirow[t]{3}{*}{20} & $\frac{1}{3}$ & 43.6850 & 50558 & 31.2769 & 44.7796 & $\underline{45.4200}$ & 45.3034 & 9 & $\underline{45.4200}$ & 45.3303 & 5 & $\underline{45.4200}$ \\
\hline & $\frac{1}{2}$ & 63.5279 & 50013 & 42.7353 & 65.647 & $\underline{71.2331}$ & 69.7546 & 10 & 70.8221 & 68.7228 & 7 & $\underline{71.2331}$ \\
\hline & $\frac{2}{3}$ & 84.7008 & 63350 & 66.9917 & 87.7261 & 95.1127 & 94.0614 & 10 & $\underline{95.2162}$ & 93.4912 & 5 & $\underline{95.2162}$ \\
\hline \multirow[t]{3}{*}{30} & $\frac{1}{3}$ & 57.9328 & 69565 & 48.1206 & 59.7641 & 66.6947 & 66.1056 & 18 & 66.6329 & 65.1248 & 14 & 66.6947 \\
\hline & $\frac{1}{2}$ & 84.3715 & 82101 & 63.4074 & 95.4395 & $\underline{100.4537}$ & 99.1734 & 20 & 100.3020 & 99.2145 & 11 & 100.4537 \\
\hline & $\frac{5}{3}$ & 110.3253 & 39564 & 88.8787 & 131.2393 & 135.2908 & 133.4534 & 20 & $\underline{137.5277}$ & 136.1031 & 12 & $\underline{137.5277}$ \\
\hline \multirow{2}{*}{\multicolumn{2}{|c|}{$\begin{array}{c}\text { Avg. dev (\%) } \\
\text { or T(s) } \\
\text { \# Best }\end{array}$}} & 9.64 & 43949 & 35.75 & 6.78 & 0.19 & 1.43 & 11 & 0.16 & 1.99 & 6 & \\
\hline & & 1 & & 0 & 0 & 7 & 1 & & 5 & & & \\
\hline
\end{tabular}

\subsubsection{Statistical significance}

The Friedman test is a non-parametric statistical procedure for comparing more than two samples that are related. When it leads to significant results, it rejects the hypothesis that there is no difference between the samples studied. Corder and Foreman (2009) provides a comprehensive introduction to this topic.

In our case, the hypotheses are the following:

$H_{0}$ (null hypothesis) : "There is no difference between the results returned by FSS, SA and VNS"

$H_{a}$ (alternative hypothesis): "There is a difference between the results returned by FSS, SA and VNS"

For every case, the number of rows is 9 (instances) and the number of columns is 3 (algorithms). The Friedman test starts by ranking the results returned by the various algorithms from 1 (for the smallest value) to 3 (for the highest one). In case of ties, equal values receive the average of their values had they 
Table 7

Results in the case of maximising the area of squares packed

\begin{tabular}{|c|c|c|c|c|c|c|c|c|c|c|c|c|}
\hline \multirow[t]{2}{*}{$n$} & \multirow[t]{2}{*}{$\rho$} & \multicolumn{2}{|c|}{ FSS } & \multicolumn{2}{|c|}{ pack } & \multicolumn{3}{|c|}{ SA } & \multicolumn{3}{|c|}{ VNS } & \multirow[b]{2}{*}{ BKS } \\
\hline & & Best & $\mathrm{T}(\mathrm{s})$ & $\operatorname{Val}\left(l_{i n c}\right)$ & $\operatorname{Val}\left(l_{d e c}\right)$ & Best & Avg & $\mathrm{T}(\mathrm{s})$ & Best & Avg & $\mathrm{T}(\mathrm{s})$ & \\
\hline \multirow[t]{3}{*}{10} & $\frac{1}{3}$ & 22.9485 & 2762 & 11.6589 & 22.4676 & 23.9878 & 23.9878 & 1 & 23.9878 & 23.3642 & 0 & 23.9878 \\
\hline & $\frac{3}{2}$ & 36.7126 & 3402 & 19.3318 & 34.1265 & 37.7471 & 37.3333 & 2 & 37.7471 & 37.3333 & 1 & 37.7471 \\
\hline & $\frac{2}{3}$ & 51.7583 & 4593 & 30.2879 & 42.9585 & $\underline{52.7555}$ & 52.7555 & 2 & $\underline{52.7555}$ & 51.9923 & 1 & $\underline{52.7555}$ \\
\hline \multirow[t]{3}{*}{20} & & 54.1054 & 9412 & 31.0113 & 56.9714 & 63.7430 & 62.9581 & 5 & $\underline{63.7523}$ & 62.7630 & 4 & $\underline{63.7523}$ \\
\hline & $\frac{1}{2}$ & 85.2107 & 11304 & 57.5113 & 87.1782 & $\underline{94.7706}$ & 94.5368 & 6 & $\underline{94.7706}$ & 94.0801 & 3 & $\underline{94.7706}$ \\
\hline & $\frac{2}{3}$ & 109.8363 & 7636 & 70.9802 & 117.5935 & 126.7480 & 126.0535 & 6 & 132.4100 & 125.8077 & 5 & 132.4100 \\
\hline \multirow[t]{3}{*}{30} & $\frac{1}{3}$ & 54.4941 & 16629 & 43.2045 & 62.4524 & 63.1167 & 62.8116 & 11 & 63.9965 & 63.4017 & 4 & $\underline{63.9965}$ \\
\hline & 2 & 77.5814 & 14808 & 65.9387 & 95.1851 & 97.2366 & 95.8948 & 12 & $\underline{98.1142}$ & 97.1655 & 12 & $\underline{98.1142}$ \\
\hline & $\frac{2}{3}$ & 103.0963 & 15145 & 87.758 & 127.511 & 129.6979 & 128.3066 & 14 & $\underline{131.5472}$ & 129.5725 & 6 & $\underline{131.5472}$ \\
\hline \multirow{2}{*}{\multicolumn{2}{|c|}{$\begin{array}{c}\text { Avg. dev (\%) } \\
\text { or T(s) } \\
\# \text { Best }\end{array}$}} & 12.07 & 9521 & 42.05 & 8.09 & 0.89 & 1.55 & 7 & 0.00 & 1.76 & 4 & \\
\hline & & 0 & & 0 & 0 & 4 & 1 & & 9 & & & \\
\hline
\end{tabular}

been different. The Friedman test statistic, denoted by $F_{r}$, is then calculated according to the following formula:

$$
F_{r}=\frac{n(k-1)\left[\sum_{i=1}^{k} \frac{R_{i}^{2}}{n}-C_{f}\right]}{\sum r_{i j}^{2}-C_{f}}
$$

where $n$ is the number of rows, $k$ is the number of columns, $R_{i}$ is the sum of the ranks from column $i$, $C_{f}$ is a ties correction factor equal to $(1 / 4) n k(k+1)^{2}$; and $r_{i j}$ is the rank corresponding to the row $i$ and column $j$.

In Table 8, the first three columns describe the case considered, the fourth column corresponds to the table taken from this paper as a basis for the calculation of $F_{r}$ and the last column is the calculated value of $F_{r}$ in the corresponding case. We consider an $\alpha$ value of 0.05 . The critical value associated with $k=3, n=9$ and $\alpha=0.05$ is 6.222 . If $F_{r}$ is greater than 6.222 , the null hypothesis is rejected in favour of the alternative hypothesis. Otherwise, there is no enough evidence to reject the null hypothesis.

In Table 8 , bold values correspond to values of $F_{r}$ greater than 6.222. The values of $F_{r}$ are greater than the critical value in five cases among six which is sufficient to reject the null hypothesis in those cases.

At this level, we conclude that in the majority of cases, we have enough statistical evidence to state that there is a difference between the results returned by the three algorithms. Together with the conclusion drawn at the previous section, we can say that the improvement made by SA and VNS over FSS is statistically significant in the majority of cases considered in the López and Beasley's dataset (2018a). 
Table 8

Friedman test statistic values for the different cases

\begin{tabular}{cccrc}
\hline Objective function $^{1}$ & Objects' $^{\prime}$ shapes $^{2}$ & Rotation angle $^{3}$ & Base Table & \multicolumn{1}{c}{$F_{r}$} \\
\hline$\#$ & $\square$ & 0 & 2 & $\mathbf{7 . 4 3}$ \\
$\#$ & $\square$ & $\frac{\pi}{2}$ & $\overline{3}$ & 4.67 \\
$\#$ & $\square$ & 0 & $\overline{\overline{4}}$ & $\mathbf{6 . 5 0}$ \\
A & $\square$ & 0 & $\overline{5}$ & $\mathbf{1 1 . 1 4}$ \\
A & $\square$ & $\frac{\pi}{2}$ & $\overline{6}$ & $\mathbf{1 3 . 0 7}$ \\
A & $\square$ & 0 & $\overline{7}$ & $\mathbf{1 6 . 7 5}$ \\
\hline
\end{tabular}

${ }^{1}$ \#: Maximise the number of objects packed, A: Maximise the area packed.

${ }^{2} \square$ : Rectangular objects, $\square$ : Square objects.

${ }^{3}$ 0: No rotation allowed, $\frac{\pi}{2}$ : Rotation by an angle of $\frac{\pi}{2}$ allowed.

\subsection{Results on the newly constructed dataset}

The four metaheuristics SA, xSA, VNS and xVNS are tested on the newly generated dataset (see Section 4.1.2). Detailed results are presented in Appendix B. Table 9 describes a summary of the results. The first three columns identify the type of instance addressed. For every metaheuristic, the columns "Dev. (\%) or Diff." and "T(s)" correspond to the average deviation or difference obtained in the considered type of instance and the average computation time (in seconds) respectively.

In terms of solution quality, the variable neighbourhood search methodologies produce the best average deviations in all cases except when maximising the number of squares packed where SA performs better. Moreover, VNS and xVNS are able together to generate 44 best solutions including ties among 54 possible ones (more than $80 \%$ ) against only 18 best solutions including ties for SA and xSA (almost 33\%). Regarding the computation time, all algorithms are able to tackle these large instances using a reasonable amount of time. The accelerated versions xSA and xVNS are in average 2.71 and 2.91 times faster than their counterparts SA and VNS though their average effectiveness is slightly affected as one may have expected (see Section 3.4). xVNS is the best performer in obtaining the best trade-off between solution quality and computation time.

\section{Two illustrative examples}

As an illustration, we present in Figure 7 the new best solution found by VNS on the instance rect3 where the objective is to maximise the area packed without rotation. In that case, FSS produced a solution with value 103.4802 in 19470 seconds whereas VNS provides a solution with value 137.4149 in 7 seconds only. Also, we present in Figure 8 the best solution found by SA in R200 where the objective is to maximise the number of rectangles packed with rotation. In that case, SA is able to pack 148 rectangles in 1519 seconds. 
Table 9

Summary results of the four metaheuristics on the newly generated dataset

\begin{tabular}{|c|c|c|c|c|c|c|c|c|c|c|}
\hline \multicolumn{3}{|c|}{ Instance type } & \multicolumn{2}{|l|}{ SA } & \multicolumn{2}{|l|}{$\mathrm{xSA}$} & \multicolumn{2}{|l|}{ VNS } & \multicolumn{2}{|c|}{$\mathrm{xVNS}$} \\
\hline $\begin{array}{c}\text { Objects' } \\
\text { shape }\end{array}$ & $\begin{array}{l}\text { Objective } \\
\text { function }\end{array}$ & $\begin{array}{c}\text { Rotation } \\
\text { angle }\end{array}$ & $\begin{array}{l}\text { Dev.(\%) or } \\
\text { Diff. }\end{array}$ & $\mathrm{T}(\mathrm{s})$ & $\begin{array}{l}\text { Dev.(\%) or } \\
\text { Diff. }\end{array}$ & $\mathrm{T}(\mathrm{s})$ & $\begin{array}{l}\text { Dev.(\%) or } \\
\text { Diff. }\end{array}$ & $\mathrm{T}(\mathrm{s})$ & $\begin{array}{c}\text { Dev.(\%) or } \\
\text { Diff. }\end{array}$ & $\mathrm{T}(\mathrm{s})$ \\
\hline$\square$ & A & 0 & 1.53 & 403 & 1.63 & 149 & 0.12 & 324 & 0.16 & 97 \\
\hline$\square$ & A & $\frac{\pi}{2}$ & 1.40 & 659 & 1.40 & 242 & 0.09 & 740 & 0.07 & 186 \\
\hline$\square$ & A & - & 1.03 & 446 & 0.95 & 153 & 0.04 & 391 & 0.12 & 102 \\
\hline$\square$ & $\#$ & 0 & 1.00 & 441 & 1.00 & 172 & 0.33 & 322 & 0.22 & 144 \\
\hline$\square$ & \# & $\frac{\pi}{2}$ & 0.89 & 692 & 1.89 & 254 & 0.33 & 601 & 0.89 & 221 \\
\hline$\square$ & $\#$ & - & $\mathbf{0 . 3 3}$ & 464 & 0.56 & 176 & 2.00 & 399 & 1.89 & 204 \\
\hline \multicolumn{3}{|c|}{ Average time (s) } & \multicolumn{2}{|l|}{517} & \multicolumn{2}{|l|}{191} & \multicolumn{2}{|l|}{463} & \multicolumn{2}{|l|}{159} \\
\hline \multicolumn{3}{|c|}{ Average deviation (\%) } & \multicolumn{2}{|l|}{1.32} & \multicolumn{2}{|l|}{1.32} & \multicolumn{2}{|l|}{0.08} & \multicolumn{2}{|l|}{0.12} \\
\hline \multicolumn{3}{|c|}{ Average difference } & \multicolumn{2}{|l|}{0.74} & \multicolumn{2}{|l|}{1.15} & 0.89 & & \multicolumn{2}{|l|}{1.00} \\
\hline \multicolumn{3}{|c|}{ Number of best solutions } & \multicolumn{4}{|c|}{18} & \multicolumn{4}{|c|}{44} \\
\hline
\end{tabular}

\section{Conclusion \& Suggestions}

In this paper, we tackled the problem of packing unequal rectangles into a circular container of fixed size with the objective of maximising the area or the number of objects packed. Given the complexity of the solution structure of the problem, we reduced our search to the space of lists of rectangles. This was possible due to a decoding procedure called pack that we introduced and which permits to convert an ordered list of rectangles into a feasible packing for the problem. This procedure which receives initially an ordered list of the objects to pack, returns a powerful packing by means of a new data structure we introduced called "border", a number of initial configurations for the packing of the first objects in the list and several powerful moves that enable an efficient packing of new objects, the whole taking advantage from the power of geometry to provide simple analytical forms. The extensions to the pack procedure we introduced widened the applications of our methodology by enabling the rotation of rectangles and forbidding connected regions in the container. As the call of pack on one single list is a constructive heuristic, we integrate pack into two metaheuristic schemes, namely, variable neighbourhood search (VNS) and simulated annealing (SA). Also, exploiting previous placements of some items in the search enabled us to provide two accelerated variants of SA and VNS denoted xSA and xVNS respectively. Experiments conducted on benchmark instances showed that our methodology is superior to the stateof-the-art method and that is able to provide 32 new best solutions out of 54 (almost $60 \%$ of the total number of instances) while requiring less computational effort. Additional experiments carried on larger instances which we constructed and also made publicly available showed that, in terms of solution quality, the VNS methodologies proposed perform better than the SA ones in most of the cases except when maximising the number of squares packed where SA does better. In terms of computation time, the 


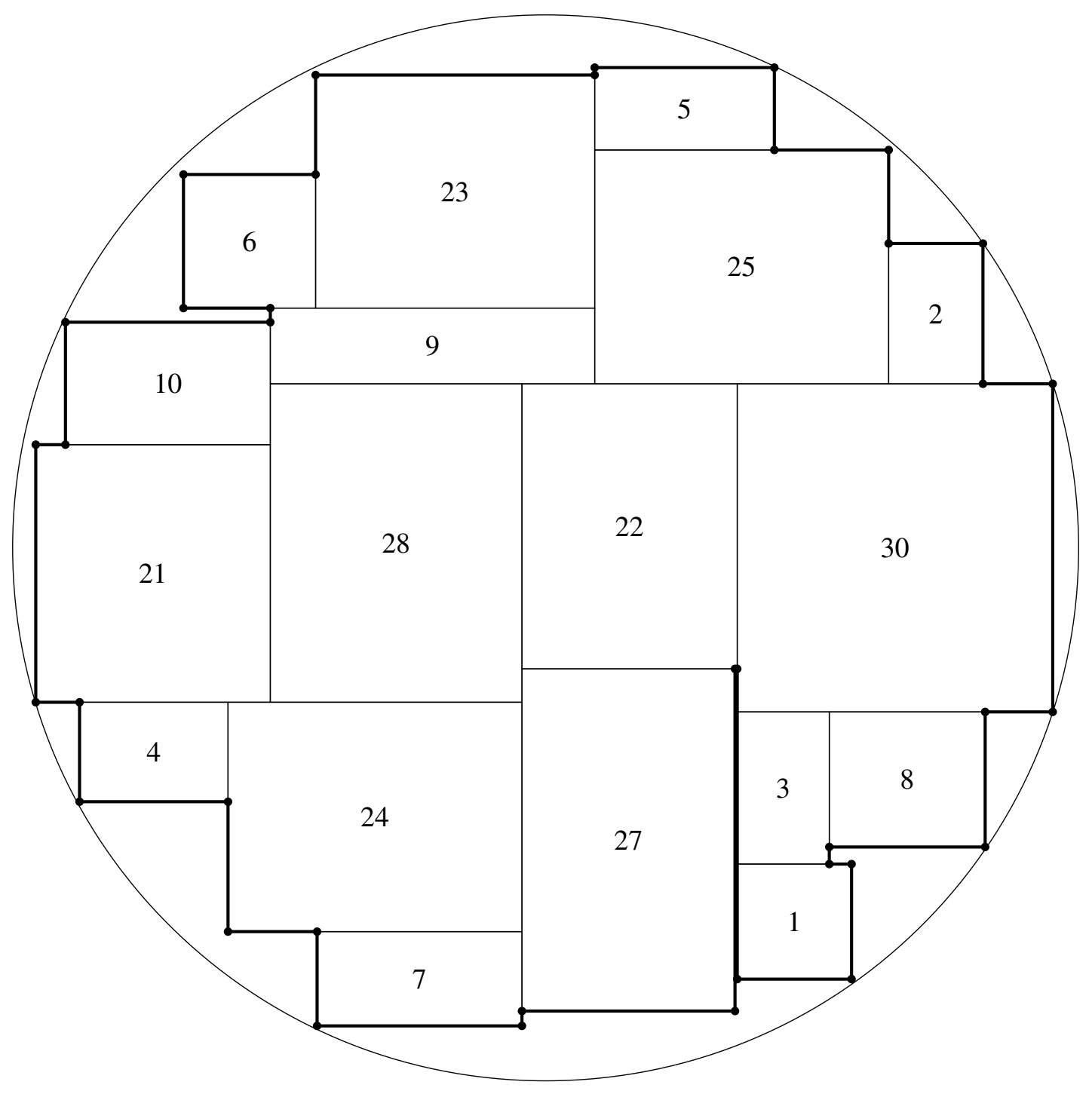

Fig. 7. Maximising the area of rectangles packed without rotation, $n=30, \rho=\frac{2}{3}$.

four metaheuristics proposed are able to tackle large instances in a reasonable amount of time. The best trade-off between solution quality and computation time is achieved by xVNS.

Among the many interesting perspectives offered by this study, we mention the possibility to extend our methodology to tackle other packing problems involving more sophisticated forms of containers and forbidden regions. The metaheuristics proposed in this paper could be easily extended by hybridising them with large neighbourhood search making them even more powerful. The exciting area of adaptive or deep learning could be explored within these metaheuristics to efficiently address these classes of packing problems and other complex global optimisation problems. 


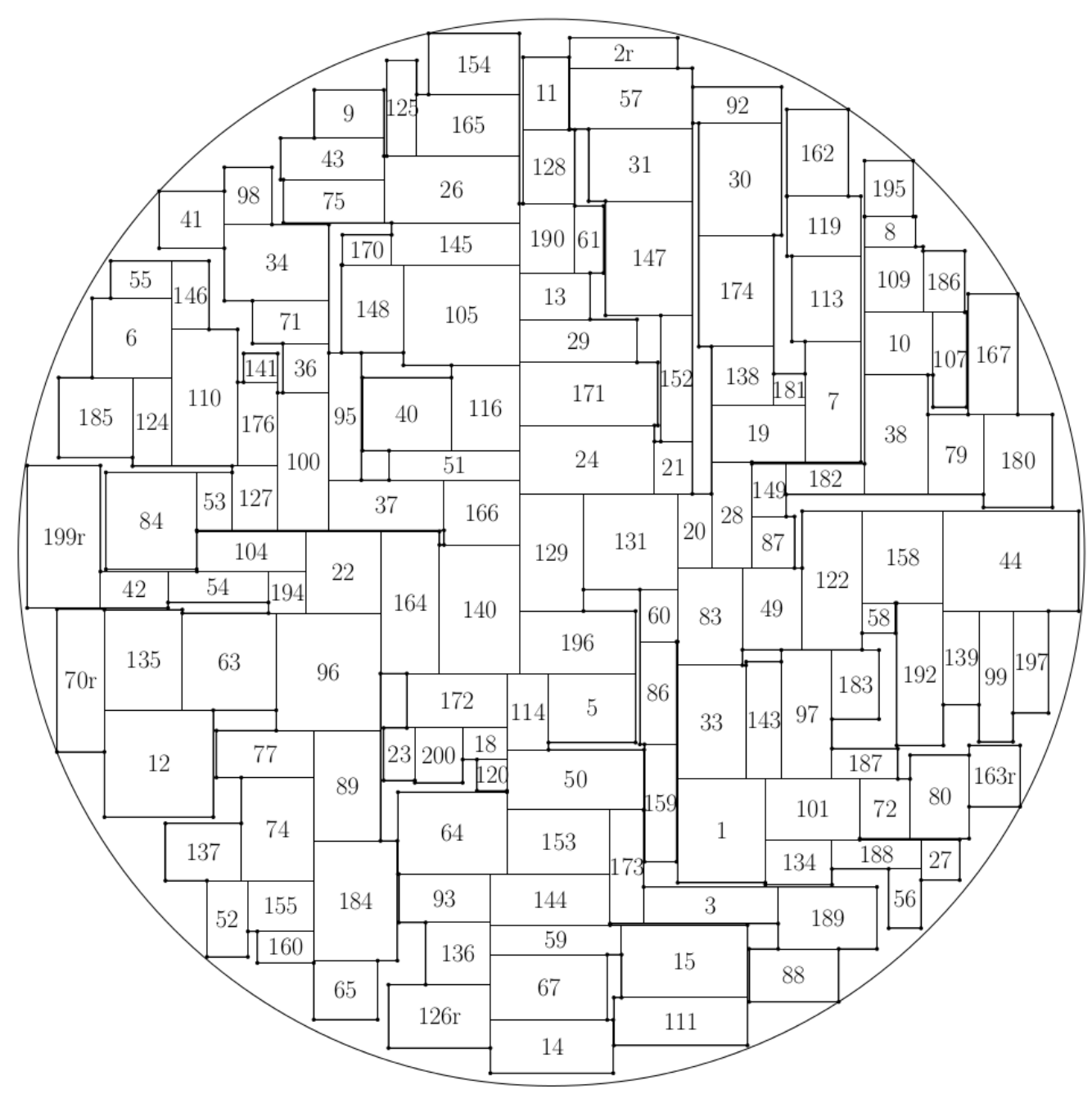

Fig. 8. Maximising the number of rectangles packed with rotation, $n=200, \rho=\frac{2}{3}$

\section{Acknowledgement}

The authors would like to thank the referees for their constructive comments that improved the presentation as well as the content of the paper.

\section{References}

Birgin, E. G. (2016). Applications of nonlinear programming to packing problems. In Applications+ Practical Conceptualization + Mathematics = fruitful Innovation, pages 31-39. Springer.

Birgin, E. G. and Lobato, R. D. (2010). Orthogonal packing of identical rectangles within isotropic convex regions. Computers \& Industrial Engineering, 59:595-602.

Birgin, E. G., Martínez, J. M., Mascarenhas, W. F., and Ronconi, D. P. (2006a). Method of sentinels for packing items within arbitrary convex regions. Journal of the Operational Research Society, 57:735746.

Birgin, E. G., Martínez, J. M., Nishihara, F. H., and Ronconi, D. P. (2006b). Orthogonal packing of 
rectangular items within arbitrary convex regions by nonlinear optimization. Computers \& Operations Research, 33:3535-3548.

Buchberger, B. (1976). A theoretical basis for the reduction of polynomials to canonical forms. ACM SIGSAM Bulletin, 10:19-29.

Cassioli, A. and Locatelli, M. (2011). A heuristic approach for packing identical rectangles in convex regions. Computers \& Operations Research, 38:1342-1350.

CLHO (2019). Centre for Logistics \& Heuristic Optimisation, University of Kent, Canterbury, UK. https://research.kent.ac.uk/clho/.

Corder, G. W. and Foreman, D. I. (2009). Nonparametric Statistics for Non-Statisticians: A Step-by-Step Approach. John Wiley \& Sons.

Demaine, E. D., Fekete, S. P., and Lang, R. J. (2010). Circle packing for origami design is hard. arXiv:1008.1224.

Dowsland, K. A. (1993). Some experiments with simulated annealing techniques for packing problems. European Journal of Operational Research, 68:389-399.

Fakoor, M., Zadeh, P. M., and Eskandari, H. M. (2017). Developing an optimal layout design of a satellite system by considering natural frequency and attitude control constraints. Aerospace Science and Technology, 71:172-188.

Feng, E., Wang, X., Wang, X., and Teng, H.-F. (1999). An algorithm of global optimization for solving layout problems. European Journal of Operational Research, 114:430-436.

Garey, M. R. and Johnson, D. S. (1979). Computers and Intractability: A Guide to the Theory of NPCompleteness. W. H. Freeman \& Co., New York, NY, USA.

Gomes, A. M. and Oliveira, J. F. (2006). Solving irregular strip packing problems by hybridising simulated annealing and linear programming. European Journal of Operational Research, 171(3):811-829.

Hansen, P., Mladenović, N., Brimberg, J., and Moreno Pérez, J. A. (2019). Variable neighborhood search. In Gendreau, M. and Potvin, J., editors, Handbook of metaheuristics, pages 57-97. Springer.

Hansen, P., Mladenović, N., and Moreno Pérez, J. A. (2010). Variable neighbourhood search: methods and applications. Annals of Operations Research, 175:367-407.

Hifi, M. and Yousef, L. (2019). A local search-based method for sphere packing problems. European Journal of Operational Research, 274:482 - 500.

Hinostroza, I., Pradenas, L., and Parada, V. (2013). Board cutting from logs: Optimal and heuristic approaches for the problem of packing rectangles in a circle. International Journal of Production Economics, 145:541-546.

Kirkpatrick, S., Gelatt, C. D., and Vecchi, M. P. (1983). Optimization by simulated annealing. Science, 220:671-680. 
Leung, J. Y.-T., Tam, T. W., Wong, C. S., Young, G. H., and Chin, F. Y. L. (1990). Packing squares into a square. Journal of Parallel and Distributed Computing, 10:271-275.

Leung, S. C. H., Zhang, D., and Sim, K. M. (2011). A two-stage intelligent search algorithm for the two-dimensional strip packing problem. European Journal of Operational Research, 215:57-69.

Leung, T. W., Chan, C. K., and Troutt, M. D. (2003). Application of a mixed simulated annealinggenetic algorithm heuristic for the two-dimensional orthogonal packing problem. European Journal of Operational Research, 145:530-542.

Li, K. and Cheng, K. H. (1994). Interconnection Networks for High-performance Parallel Computers, chapter Complexity of Resource Allocation and Job Scheduling Problems in Partitionable Mesh Connected Systems, pages 644-651. IEEE Computer Society Press, Los Alamitos, CA, USA.

Li, Z., Wang, X., Tan, J., and Wang, Y. (2014). A quasiphysical and dynamic adjustment approach for packing the orthogonal unequal rectangles in a circle with a mass balance: satellite payload packing. Mathematical Problems in Engineering, 2014.

Li, Z., Zeng, Y., Wang, Y., Wang, L., and Song, B. (2016). A hybrid multi-mechanism optimization approach for the payload packing design of a satellite module. Applied Soft Computing, 45:11-26.

Liu, D. C. and Nocedal, J. (1989). On the limited memory BFGS method for large scale optimization. Mathematical programming, 45:503-528.

Liu, Z. and Teng, H.-F. (2008). Human-computer cooperative layout design method and its application. Computers \& Industrial Engineering, 55:735-757.

López, C. O. and Beasley, J. E. (2011). A heuristic for the circle packing problem with a variety of containers. European Journal of Operational Research, 214:512-525.

López, C. O. and Beasley, J. E. (2013). Packing unequal circles using formulation space search. Computers \& Operations Research, 40:1276-1288.

López, C. O. and Beasley, J. E. (2016). A formulation space search heuristic for packing unequal circles in a fixed size circular container. European Journal of Operational Research, 251:64-73.

López, C. O. and Beasley, J. E. (2018a). Dataset for the packing of unequal rectangles into a circular container. http://people.brunel.ac.uk/ mastjjb/jeb/orlib/files/ Accessed 15 July 2018.

López, C. O. and Beasley, J. E. (2018b). Packing unequal rectangles and squares in a fixed size circular container using formulation space search. Computers \& Operations Research, 94:106 - 117.

Martins, T. C. and Tsuzuki, M. S. G. (2010). Simulated annealing applied to the irregular rotational placement of shapes over containers with fixed dimensions. Expert Systems with Applications, 37:19551972 .

M'Hallah, R. and Alkandari, A. (2012). Packing unit spheres into a cube using VNS. Electronic Notes in Discrete Mathematics, 39:201-208. 
M'Hallah, R., Alkandari, A., and Mladenović, N. (2013). Packing unit spheres into the smallest sphere using VNS and NLP. Computers \& Operations Research, 40:603-615.

Mladenović, N. and Hansen, P. (1997). Variable neighborhood search. Computers \& Operations Research, 24:1097 - 1100 .

Mladenović, N., Plastria, F., and Urošević, D. (2005). Reformulation descent applied to circle packing problems. Computers \& Operations Research, 32:2419-2434.

Mladenović, N., Plastria, F., and Urošević, D. (2007). Formulation space search for circle packing problems. In Stützle, T., Birattari, M., and Hoos, H., editors, Engineering Stochastic Local Search Algorithms. Designing, Implementing and Analyzing Effective Heuristics, pages 212-216, Berlin, Heidelberg. Springer Berlin Heidelberg.

PassMark (2020). CPU Comparison Intel i3-2330M vs Intel i5-2400S. https://www.cpubenchmark.net/compare/Intel-i3-2330M-vs-Intel-i5-2400S/757vs794 Accessed 7 January 2020.

Prins, C., Lacomme, P., and Prodhon, C. (2014). Order-first split-second methods for vehicle routing problems: A review. Transportation Research Part C, 40:179-200.

SageMath, Inc. (2018). Cocalc collaborative computation online. https://cocalc.com/ Accessed 27 November 2018.

Salhi, S. (2017). Heuristic Search: The Emerging Science of Problem Solving. Springer.

Soke, A. and Bingul, Z. (2006). Hybrid genetic algorithm and simulated annealing for two-dimensional non-guillotine rectangular packing problems. Engineering Applications of Artificial Intelligence, 19:557 - 567 .

Sun, Z.-G. and Teng, H.-F. (2003). Optimal layout design of a satellite module. Engineering Optimization, 35:513-529.

Talbi, E.-G. (2009). Metaheuristics: from design to implementation. John Wiley \& Sons.

Teng, H.-F., Sun, S., Liu, D., and Li, Y. (2001). Layout optimization for the objects located within a rotating vessel - a three-dimensional packing problem with behavioral constraints. Computers \& Operations Research, 28:521-535.

Wang, Y. and Teng, H.-F. (2009). Knowledge fusion design method: satellite module layout. Chinese Journal of Aeronautics, 22:32-42.

Wäscher, G., Haußner, H., and Schumann, H. (2007). An improved typology of cutting and packing problems. European Journal of Operational Research, 183:1109 - 1130.

Xu, Y., Xiao, R., and Amos, M. (2007). Particle swarm algorithm for weighted rectangle placement. In Third International Conference on Natural Computation, 2007 (ICNC 2007), pages 728-732. IEEE.

Xu, Y.-C., Dong, F.-M., Liu, Y., and Xiao, R.-B. (2010). Genetic algorithm for rectangle layout optimization with equilibrium constraints. Pattern Recognition and Artificial Intelligence, 23:794-801. 
Zeng, Z., Yu, X., He, K., and Fu, Z. (2018). Adaptive tabu search and variable neighborhood descent for packing unequal circles into a square. Applied Soft Computing, 65:196-213.

Zeng, Z., Yu, X., He, K., Huang, W., and Fu, Z. (2016). Iterated tabu search and variable neighborhood descent for packing unequal circles into a circular container. European Journal of Operational Research, 250:615-627.

Zhang, B., Teng, H.-F., and Shi, Y.-J. (2008). Layout optimization of satellite module using soft computing techniques. Applied Soft Computing, 8:507-521.

Zhao, F., Li, G., Yang, C., Abraham, A., and Liu, H. (2014). A human-computer cooperative particle swarm optimization based immune algorithm for layout design. Neurocomputing, 132:68-78.

Zhong, C.-Q., Xu, Z.-Z., and Teng, H.-F. (2019). Multi-module satellite component assignment and layout optimization. Applied Soft Computing, 75:148-161. 


\section{A Complementary data for the calibration of VNS and SA}

Prior to the experiments conducted in Sections 4.2 and 4.3, we carried out a parameter calibration of our algorithms. This was conducted on the instance R100 that we generated and which is available at the CLHO (2019) website. The problem considered consists in maximising the area packed by 100 candidate rectangles into a circular container of radius 14.14 with rotation. Tables 10 and 11 present the results obtained during the calibration of VNS and SA respectively. In Table 10, the first two columns correspond to the combinations of parameter values tested while the two last columns are the average solution values and the average computing times in seconds over 5 runs respectively. The results presented in Table 11 correspond to the 15 combinations of parameters values which brought the best average solution values among 64 combinations tested. The first six columns correspond to the combinations tested while the last two columns are the average solution values and average computing times in seconds over 3 runs respectively. In both tables, results are sorted in the decreasing order of the average solution value obtained. Bold rows correspond to the selected combinations of parameters values.

\section{Table 10}

Results obtained during the calibration of VNS

\begin{tabular}{ccrr}
\hline$k_{\max }$ & $i t_{\max }$ & Avg. Value & Avg. Time (s) \\
\hline $\mathbf{3}$ & $\mathbf{5 \times \mathbf { 1 0 } ^ { \mathbf { 2 } }}$ & $\mathbf{5 6 8 . 9 4 7 0}$ & $\mathbf{5 1}$ \\
3 & $10^{3}$ & 568.8626 & 112 \\
5 & $10^{3}$ & 568.3557 & 86 \\
5 & $5 \times 10^{2}$ & 567.1563 & 58 \\
7 & $10^{3}$ & 566.9445 & 91 \\
7 & $5 \times 10^{2}$ & 564.6677 & 42 \\
\hline
\end{tabular}

Table 11

Results obtained during the calibration of SA (15 best)

\begin{tabular}{rcccccrr}
\hline$T_{0}$ & $T_{f}$ & $\alpha$ & $N_{t}$ & $k$ & $i t_{\max }$ & Avg. Value & Time (s) \\
\hline $\mathbf{1 0}$ & $\mathbf{0 . 1}$ & $\mathbf{0 . 9 8}$ & $\mathbf{5}$ & $\mathbf{3}$ & $\mathbf{1 0}^{\mathbf{4}}$ & $\mathbf{5 6 2 . 1 6 9 3}$ & $\mathbf{7 1}$ \\
10 & 0.1 & 0.98 & 10 & 3 & $10^{4}$ & 561.2068 & 158 \\
20 & 0.1 & 0.98 & 10 & 3 & $10^{3}$ & 560.7677 & 50 \\
20 & 0.1 & 0.95 & 5 & 3 & $10^{3}$ & 560.0849 & 26 \\
20 & 0.01 & 0.98 & 10 & 3 & $10^{3}$ & 559.8920 & 62 \\
20 & 0.1 & 0.95 & 10 & 3 & $10^{3}$ & 559.3519 & 47 \\
10 & 0.1 & 0.95 & 10 & 3 & $10^{4}$ & 559.3456 & 62 \\
10 & 0.01 & 0.95 & 5 & 3 & $10^{4}$ & 559.0968 & 40 \\
10 & 0.1 & 0.95 & 5 & 3 & $10^{4}$ & 558.8763 & 30 \\
10 & 0.01 & 0.98 & 5 & 3 & $10^{4}$ & 558.8584 & 106 \\
10 & 0.01 & 0.98 & 5 & 3 & $10^{3}$ & 558.7867 & 57 \\
10 & 0.01 & 0.95 & 10 & 3 & $10^{4}$ & 558.7666 & 89 \\
10 & 0.01 & 0.95 & 10 & 3 & $10^{3}$ & 558.6839 & 55 \\
10 & 0.1 & 0.95 & 5 & 7 & $10^{3}$ & 558.6774 & 31 \\
10 & 0.1 & 0.95 & 5 & 3 & $10^{3}$ & 558.4810 & 27 \\
\hline
\end{tabular}




\section{B Detailed results on the newly generated dataset}

Tables 12,17 present the results obtained by SA, xSA, VNS and xVNS on the dataset we have generated (see Section 4.1.2). The tables follow the same structure, that is, the first two columns identify the instance and the next columns present the results obtained by applying five runs of SA, xSA, VNS and $\mathrm{xVNS}$ respectively. The times presented in those columns correspond to the total time in seconds spent by each algorithm over the five runs for every instance. Bold values correspond to the best solution value found. The last rows provide average measures over the instances and the number of best values obtained.

Table 12

Results in the case of maximising the number of rectangles packed without rotation

\begin{tabular}{|c|c|c|c|c|c|c|c|c|c|c|c|c|c|c|}
\hline \multirow[t]{2}{*}{$n$} & \multirow[t]{2}{*}{$\rho$} & \multicolumn{3}{|c|}{ SA } & \multicolumn{3}{|c|}{ xSA } & \multicolumn{3}{|c|}{ VNS } & \multicolumn{3}{|c|}{$\mathrm{xVNS}$} & \multirow[b]{2}{*}{ BKS } \\
\hline & & Best & Avg. & $\mathrm{T}(\mathrm{s})$ & Best & Avg. & $\mathrm{T}(\mathrm{s})$ & Best & Avg. & $\mathrm{T}(\mathrm{s})$ & Best & Avg. & $\mathrm{T}(\mathrm{s})$ & \\
\hline \multirow[t]{3}{*}{100} & $\frac{1}{3}$ & 45 & 44.20 & 101 & 45 & 43.80 & 33 & 45 & 44.00 & 66 & 44 & 43.20 & 29 & 45 \\
\hline & $\frac{1}{2}$ & 59 & 57.60 & 141 & 58 & 57.60 & 49 & 58 & 57.60 & 85 & 59 & 58.00 & 43 & 59 \\
\hline & $\frac{2}{3}$ & 70 & 69.80 & 181 & 70 & 69.80 & 64 & 69 & 69.00 & 95 & 70 & 69.60 & 51 & 70 \\
\hline \multirow[t]{3}{*}{150} & $\frac{1}{3}$ & 70 & 68.80 & 272 & 69 & 68.20 & 96 & 72 & 71.20 & 217 & 72 & 71.20 & 84 & 72 \\
\hline & $\frac{1}{2}$ & 91 & 90.40 & 380 & 91 & 90.40 & 156 & 92 & 91.80 & 258 & 91 & 90.60 & 118 & 92 \\
\hline & $\frac{2}{3}$ & 110 & 108.80 & 501 & 110 & 108.60 & 208 & 110 & 108.40 & 286 & 110 & 108.40 & 175 & 110 \\
\hline \multirow[t]{3}{*}{200} & $\frac{1}{3}$ & 95 & 94.00 & 534 & 96 & 94.40 & 212 & 99 & 97.00 & 468 & 99 & 97.40 & 221 & 99 \\
\hline & $\frac{1}{2}$ & 123 & 121.80 & 818 & 124 & 121.80 & 317 & 124 & 123.20 & 737 & 124 & 122.80 & 244 & 124 \\
\hline & $\frac{2}{3}$ & 146 & 144.80 & 1038 & 146 & 144.40 & 411 & 146 & 145.20 & 683 & 147 & 145.20 & 334 & 147 \\
\hline \multirow{2}{*}{\multicolumn{2}{|c|}{$\begin{array}{c}\text { Avg. diff. or T(s) } \\
\text { \# Best }\end{array}$}} & 1.00 & 1.98 & 441 & 1.00 & 2.11 & 172 & 0.33 & 1.18 & 322 & 0.22 & 1.29 & 144 & \\
\hline & & 4 & & & 4 & & & 6 & & & 7 & & & \\
\hline
\end{tabular}

Table 13

Results in the case of maximising the number of rectangles packed with rotation

\begin{tabular}{|c|c|c|c|c|c|c|c|c|c|c|c|c|c|c|}
\hline \multirow[t]{2}{*}{$n$} & \multirow[t]{2}{*}{$\rho$} & \multicolumn{3}{|c|}{ SA } & \multicolumn{3}{|c|}{$\mathrm{xSA}$} & \multicolumn{3}{|c|}{ VNS } & \multicolumn{3}{|c|}{ xVNS } & \multirow[b]{2}{*}{ BKS } \\
\hline & & Best & Avg. & $\mathrm{T}(\mathrm{s})$ & Best & Avg. & $\mathrm{T}(\mathrm{s})$ & Best & Avg. & $\mathrm{T}(\mathrm{s})$ & Best & Avg. & $\mathrm{T}(\mathrm{s})$ & \\
\hline \multirow[t]{3}{*}{100} & $\frac{1}{3}$ & 46 & 45.2 & 185 & 45 & 43.8 & 56 & 45 & 44.4 & 110 & 44 & 43.8 & 44 & 46 \\
\hline & $\frac{1}{2}$ & 59 & 58.0 & 239 & 59 & 58.2 & 79 & 59 & 57.8 & 164 & 59 & 58.2 & 69 & 59 \\
\hline & $\frac{2}{3}$ & 71 & 70.4 & 275 & 71 & 69.8 & 97 & 70 & 69.4 & 149 & 70 & 69.0 & 65 & 71 \\
\hline \multirow[t]{3}{*}{150} & $\frac{1}{3}$ & 70 & 69.4 & 458 & 69 & 68.8 & 167 & 73 & 71.6 & 463 & 72 & 71.2 & 120 & 73 \\
\hline & 2 & 92 & 90.6 & 611 & 90 & 90.0 & 229 & 93 & 91.8 & 379 & 92 & 91.4 & 175 & 93 \\
\hline & $\frac{2}{3}$ & 110 & 109.0 & 745 & 109 & 108.0 & 287 & 110 & 108.8 & 570 & 109 & 108.4 & 209 & 110 \\
\hline \multirow[t]{3}{*}{200} & $\frac{1}{3}$ & 95 & 94.6 & 927 & 95 & 94.4 & 321 & 98 & 97.4 & 805 & 98 & 97.0 & 267 & 98 \\
\hline & $\frac{1}{2}$ & 125 & 122.6 & 1267 & 122 & 121.2 & 469 & 126 & 124.4 & 1190 & 125 & 123.8 & 593 & 126 \\
\hline & $\frac{2}{3}$ & 148 & 146.0 & 1519 & 147 & 146.4 & 582 & 147 & 146.4 & 1579 & 147 & 145.8 & 451 & 148 \\
\hline \multirow[t]{2}{*}{ Avg } & or $\mathrm{T}(\mathrm{s})$ & 0.89 & 2.02 & 692 & 1.89 & 2.60 & 254 & 0.33 & 1.33 & 601 & 0.89 & 1.71 & 221 & \\
\hline & & 5 & & & 2 & & & 6 & & & 2 & & & \\
\hline
\end{tabular}




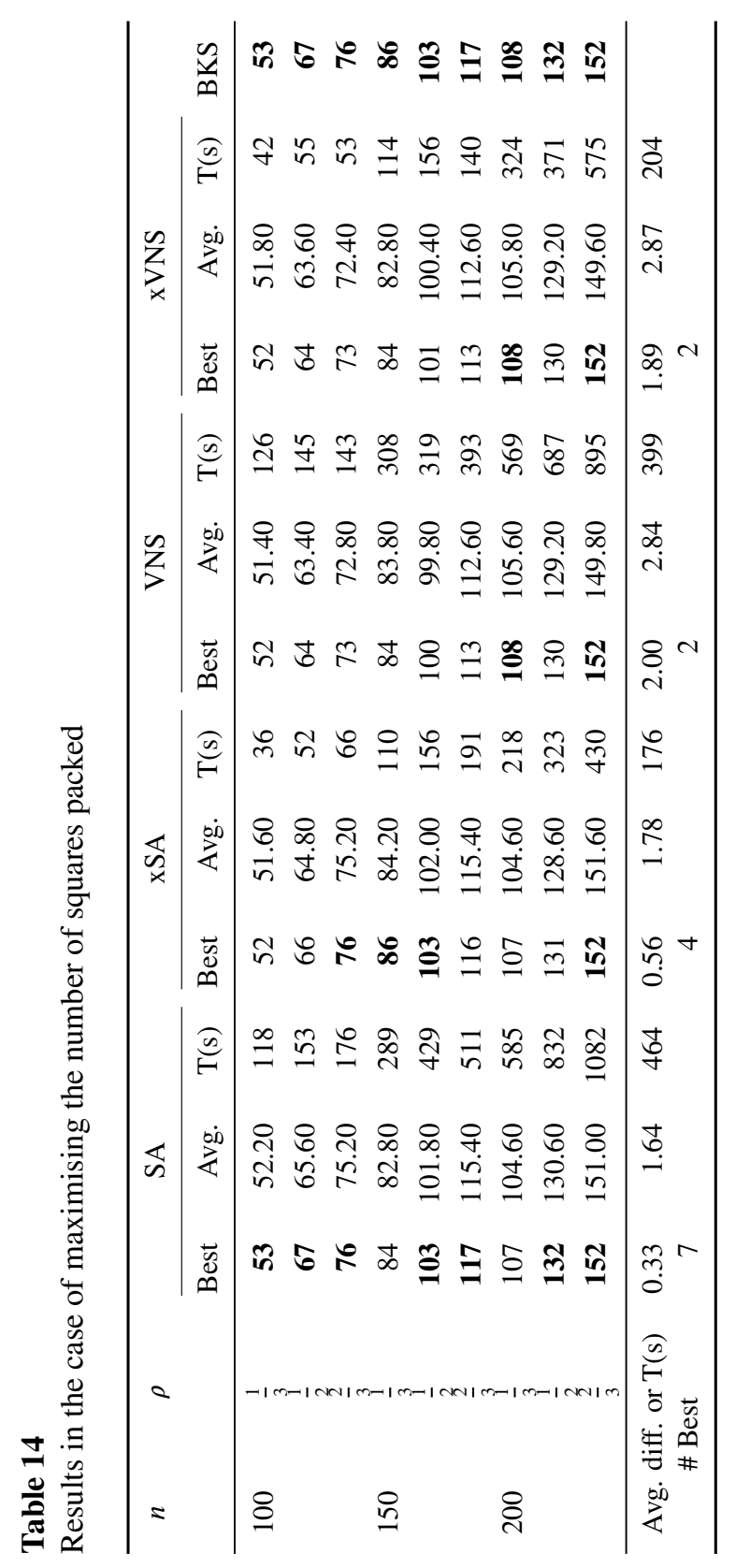



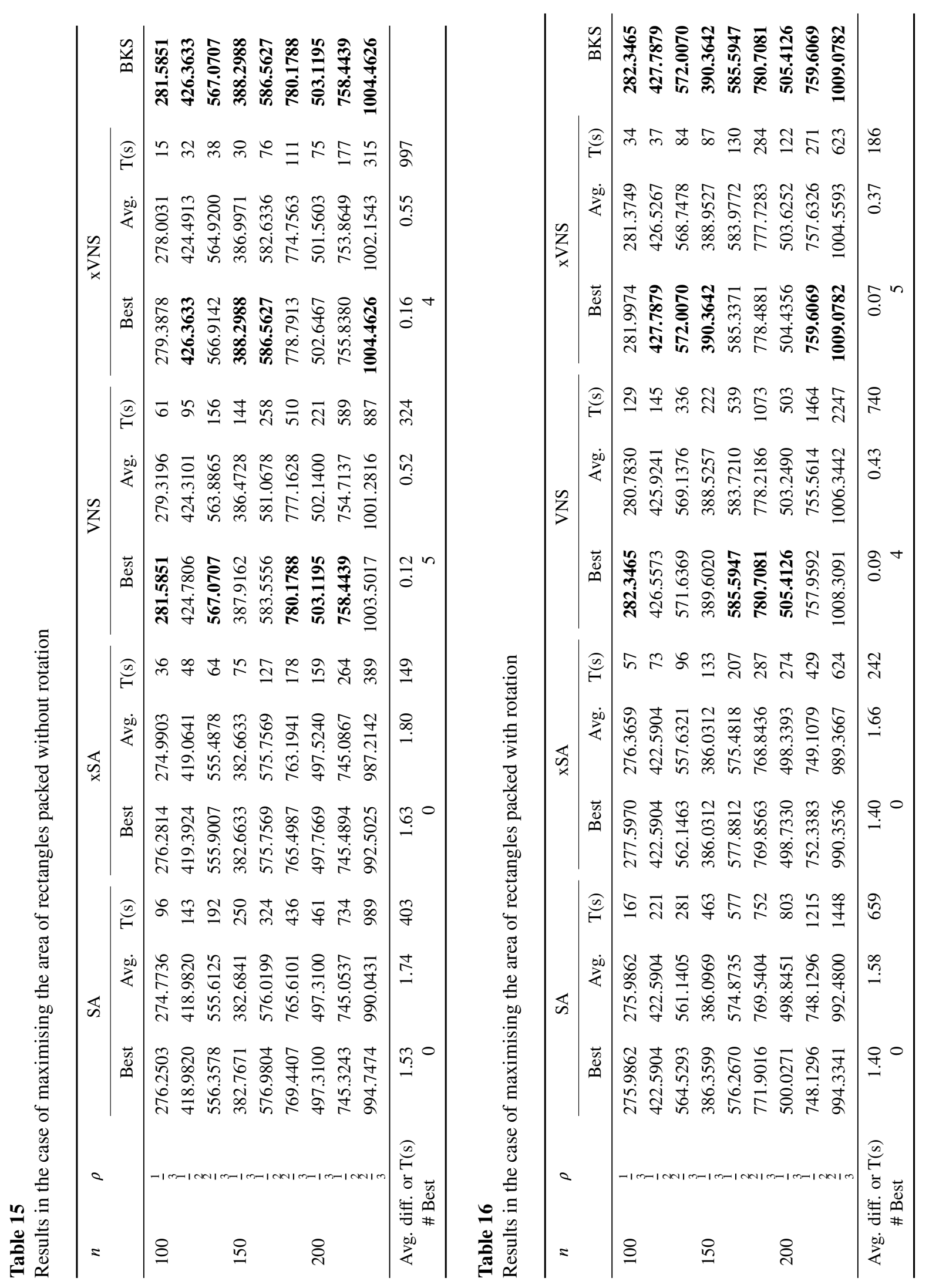


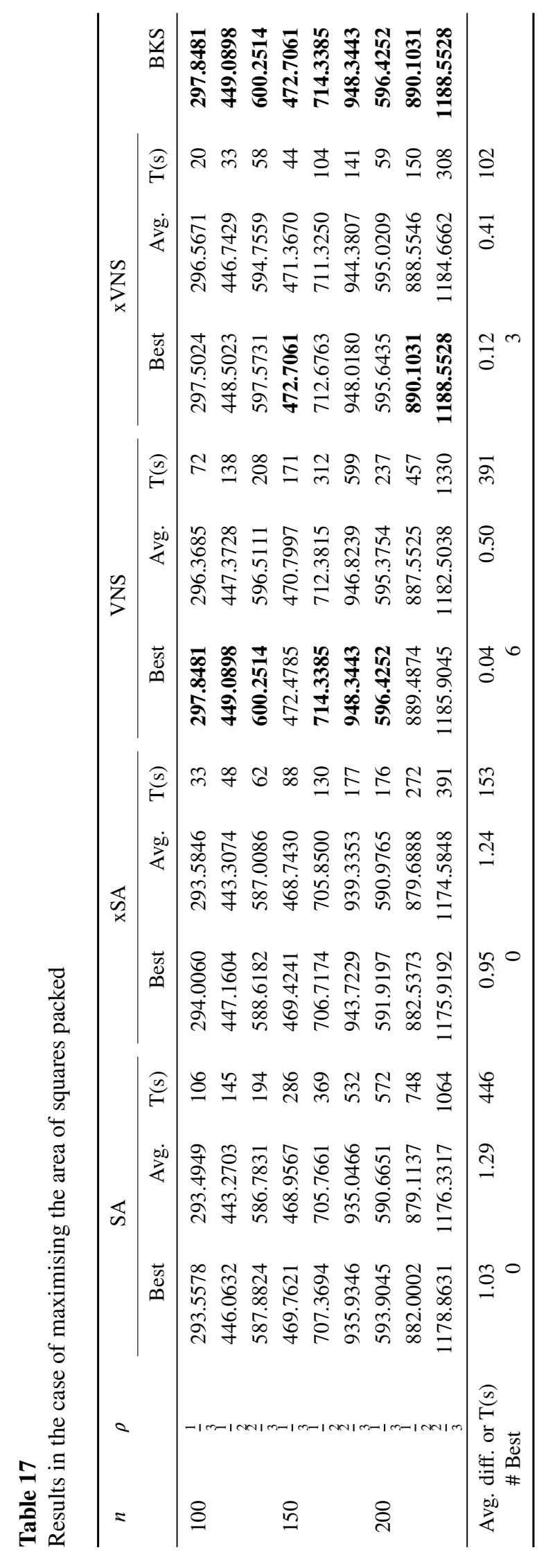

